\title{
New Delay-Dependent Exponential Stability Criteria for Neural Networks with Mixed Time-Varying Delays
}

\author{
Wu Wen ${ }^{1}$ and Kaibo Shi ${ }^{2,3}$ \\ ${ }^{1}$ Department of Academic Affairs Office, Sichuan University of Arts and Science of China, Dazhou 635000, China \\ ${ }^{2}$ School of Automation Engineering, University of Electronic Science and Technology of China, Chengdu 611731, China \\ ${ }^{3}$ Department of Applied Mathematics, University of Waterloo, Waterloo, ON, Canada N2L 3G1
}

Correspondence should be addressed to Wu Wen; dzwenwu@163.com

Received 17 December 2014; Revised 8 April 2015; Accepted 15 April 2015

Academic Editor: Asier Ibeas

Copyright (c) $2015 \mathrm{~W}$. Wen and K. Shi. This is an open access article distributed under the Creative Commons Attribution License, which permits unrestricted use, distribution, and reproduction in any medium, provided the original work is properly cited.

This study is concerned with the problem of new delay-dependent exponential stability criteria for neural networks (NNs) with mixed time-varying delays via introducing a novel integral inequality approach. Specifically, first, by taking fully the relationship between the terms in the Leibniz-Newton formula into account, several improved delay-dependent exponential stability criteria are obtained in terms of linear matrix inequalities (LMIs). Second, together with some effective mathematical techniques and a convex optimization approach, less conservative conditions are derived by constructing an appropriate Lyapunov-Krasovskii functional (LKF). Third, the proposed methods include the least numbers of decision variables while keeping the validity of the obtained results. Finally, three numerical examples with simulations are presented to illustrate the validity and advantages of the theoretical results.

\section{Introduction}

Over the course of the past decade, neural networks have become an important area of research and attracted increasing attention due to their extensive applications in many practical systems, such as power systems [1], pattern recognition [2], signal detection [3], landmark recognition [4], and other scientific areas [5-7].

Moreover, it is inevitable to introduce time delay into the signals transmitted among neurons because the processes of transcription and translation are not instantaneous. However, it is a well-known fact that time delay as a source of instability and poor performance usually appears in many dynamical systems, for instance, Cohen-Grossberg neural networks, cellular neural networks, BAM neural network, chaotic neural networks, $H_{\infty}$ filtering, and nonlinear systems [8-53]. Therefore, stability analysis for neural networks with delays has been an attractive subject of research in recent years [8-42, 50-53].

Furthermore, neural networks (NNs) often have a spatial nature due to the presence of many parallel pathways of a variety of axon sizes and lengths. Thus, in order to have a more accurate model, a distributed delay over a certain time of duration needs to be included in NNs such that the distant past has less influence compared to the recent behavior of the state. Therefore, there has been a growing interest in the study of neural networks with discrete and distributed delays during the past two decades. To date, some results on delay-dependent exponential stability for neural networks with mixed time-varying delays have been reported in $[18-23,34-36]$. In [18], the authors considered the global asymptotic stability for a class of delayed cellular neural networks with mixed time-varying delays by using LMIs approach, Lyapunov theory, and Leibniz-Newton formula. However, the activation functions in [18] were assumed to be monotonically nondecreasing. In [19], several delaydependent sufficient conditions are obtained to guarantee the global asymptotic and exponential stability of the addressed neural networks by employing appropriate LKF and linear matrix inequality (LMI) technique. In [20], an exponential stability criterion is proposed by constructing an augmented LKF, where the discrete delay $d(t)$ must be differentiable. In $[22,23]$, some improved delay-dependent stability criteria are derived in terms of linear matrix inequalities by dividing 
the discrete delay interval into multiple segments. Different from [35], the appropriate LKF not only divides the discrete delay interval $[0, d]$ into two ones $[0, d / 2]$ and $[d / 2, d]$, but also divides the discrete delay interval $[0, d]$ into three ones $[0, d(t) / 2],[d(t) / 2, d(t)]$, and $[d(t), d]$. Although this approach seems to be effective for achieving less conservative conditions, it can increase the larger numbers of computed variables. Hence, there exists great room for further improvement. To the best of our knowledge, it is of a great significance for the current research to find a more effective approach to get rid of the strict constraint and obtain less conservative conditions.

Motivated by the above discussion, combining effective mathematical techniques and a convex optimization approach, we choose a more general type of LKF to study the delay-dependent exponential stability criteria for neural networks (NNs) with mixed time-varying delays in the paper. Some improved delay-dependent stability conditions derived benefit mainly from using firstly a new integral inequality approach, which is proved to be less conservative than the celebrated Jensen's inequality and showed having a great potential efficient in practice. Both theoretical and numerical comparisons have been provided to show the effectiveness and efficiency of the proposed method. Besides, the main merit of this method lies in containing the least numbers of decision variables while keeping the validity of the obtained results. Finally, the stability criteria obtained turn out to be less conservative than some recently reported ones via three numerical examples.

Notation. Notations used in this paper are fairly standard: $\mathbb{R}^{n}$ denotes the $n$-dimensional Euclidean space, and $\mathbb{R}^{n \times m}$ is the set of all $n \times m$ dimensional matrices; $I$ is the identity matrix of appropriate dimensions, and $A_{T}$ is the matrix transposition of the matrix $A$. By $X>0$ (resp., $X \geq 0$ ), for $X \in$ $\mathbb{R}^{n \times n}$, we mean that the matrix $X$ is real symmetric positive definite (resp., positive semidefinite); $\operatorname{diag}\left\{r_{1}, r_{2}, \ldots, r_{n}\right\}$ denotes block diagonal matrix with diagonal elements $r_{i}, i=$ $1,2, \ldots, n$, and the symbol $*$ represents the elements below the main diagonal of a symmetric matrix; $\vec{A}$ is defined as $\vec{A}=A+A^{T}$.

\section{Preliminaries}

Consider the following neural networks with mixed timevarying delays:

$$
\begin{aligned}
\dot{u}(t)= & -A_{0} u(t)+A_{1} g(u(t))+A_{2} g(u(t-h(t))) \\
& +A_{3} \int_{t-r}^{t} g(u(s)) d s+I,
\end{aligned}
$$

where $u(t)=\left[u_{1}(t), u_{2}(t), \ldots, u_{n}(t)\right]^{T} \in \mathbb{R}^{n}$ is the neural state vector and $g(u(t))=\left[g_{1}\left(u_{1}(t)\right), g_{2}\left(u_{2}(t)\right), \ldots, g_{n}\left(u_{n}(t)\right)\right]^{T} \epsilon$ $\mathbb{R}^{n}$ is the neuron activation function; $I=\left[I_{1}, I_{2}, \ldots, I_{n}\right]^{T} \in$ $\mathbb{R}^{n}$ is an external constant input vector, $A_{0}=$ $\operatorname{diag}\left\{a_{01}, a_{02}, \ldots, a_{0 n}\right\}>0$, and $A_{1}, A_{2}$, and $A_{3}$ are the constant matrices of appropriate dimensions.
Assumption A. The time-varying delay $h(t)$ is continuous and differential function satisfying

$$
\begin{aligned}
0 & \leq h(t) \leq h, \\
h(t) & \leq h_{D}<1 .
\end{aligned}
$$

Assumption B. For the constants $l_{i}^{-}$and $l_{i}^{+}$the bounded activation function $g_{i}(\cdot)$ in (1) satisfies the following condition:

$$
\begin{aligned}
l_{i}^{-} \leq \frac{g_{i}(\alpha)-g_{i}(\beta)}{\alpha-\beta} & \leq l_{i}^{+}, \\
& \forall \alpha, \beta \in \mathbb{R}, \alpha \neq \beta, i=1,2, \ldots, n .
\end{aligned}
$$

We denote $L^{+}=\operatorname{diag}\left\{l_{1}^{+}, l_{2}^{+}, \ldots, l_{n}^{+}\right\}, L^{-}=\operatorname{diag}\left\{l_{1}^{-}, l_{2}^{-}\right.$, $\left.\ldots, l_{n}^{-}\right\}, L_{1}=\operatorname{diag}\left\{l_{1}^{+} l_{1}^{-}, l_{1}^{+} l_{2}^{-}, \ldots, l_{n}^{+} l_{n}^{-}\right\}, L_{2}=\operatorname{diag}\left\{\left(l_{1}^{+}+\right.\right.$ $\left.\left.l_{1}^{-}\right) / 2,\left(l_{2}^{+}+l_{2}^{-}\right) / 2, \ldots,\left(l_{n}^{+}+l_{n}^{-}\right) / 2\right\}$, and $L=\operatorname{diag}\left\{\max \left\{\left|l_{1}^{+}\right|\right.\right.$, $\left.\left.\left|l_{1}^{-}\right|\right\}, \ldots, \max \left\{\left|l_{n}^{+}\right|,\left|l_{n}^{-}\right|\right\}\right\}=\operatorname{diag}\left\{l_{1}, \ldots, l_{n}\right\}$.

Under Assumption B, by using Brouwer's fixed-point theorem [25], it can be easily proven that there exists one equilibrium point for system (1). Assuming that $u^{*}=$ $\left[u_{1}^{*}, \ldots, u_{n}^{*}\right]^{T}$ is an equilibrium point of system (1). For convenience, we firstly shift the equilibrium point $u^{*}$ to the origin by letting $z(t)=u(t)-u^{*}$ and $f(z(t))=g(u(t))-g\left(u^{*}\right)$, and then system (1) can be converted to

$$
\begin{aligned}
\dot{z}(t)= & -A_{0} z(t)+A_{1} f(z(t))+A_{2} f(z(t-h(t))) \\
& +A_{3} \int_{t-r}^{t} f(z(s)) d s,
\end{aligned}
$$

where $f(x(t))=\left[f_{1}\left(z_{1}\right), f_{2}\left(z_{2}\right), \ldots, f_{n}\left(z_{n}\right)\right]^{T}$. It is easy to check that the function $f_{i}(\cdot)$ satisfies $f_{i}(0)=0$, and

$$
l_{i}^{-} \leq \frac{f_{i}(\alpha)}{\alpha} \leq l_{i}^{+}, \quad \forall \alpha \in \mathbb{R}, \alpha \neq 0, i=1,2, \ldots, n .
$$

Due to the influence of external factors, $\int_{t-r}^{t} f(z(s)) d s$ cannot express the actual state of the accurate information. Therefore, by translating $r$ to function $r(t)(0 \leq r(t) \leq r)$, we have

$$
\begin{aligned}
\dot{z}(t)= & -A_{0} z(t)+A_{1} f(z(t))+A_{2} f(z(t-h(t))) \\
& +A_{3} \int_{t-r(t)}^{t} f(z(s)) d s .
\end{aligned}
$$

In the paper, we will attempt to formulate some practically computable criteria to check the global exponential stability of system (6). The following lemmas are useful in deriving the criteria.

Definition 1 (see [22]). The equilibrium point 0 of system (6) is said to be globally exponentially stable, if there exist scalars $k>0$ and $\beta>0$ such that

$$
\|z(t)\| \leq \beta e^{-k t} \sup _{-h \leq s \leq 0}\|z(s)\|, \quad \forall t>0 .
$$


Lemma 2 (see [22]). The following inequalities are true:

$$
\begin{aligned}
& 0 \leq \int_{0}^{z_{i}(t)}\left(f_{i}(s)-l_{i}^{-} s\right) d s \leq\left(f_{i}\left(z_{i}(t)\right)-l_{i}^{-} z_{i}(t)\right) z_{i}(t), \\
& 0 \leq \int_{0}^{z_{i}(t)}\left(l_{i}^{+} s-f_{i}(s)\right) d s \leq\left(l_{i}^{+} z_{i}(t)-f_{i}\left(z_{i}(t)\right)\right) z_{i}(t) .
\end{aligned}
$$

Lemma 3 (see [24]). For any positive definite matrix $\left[\begin{array}{cc}S & G \\ G^{T} & S\end{array}\right]>$ 0 and $r \geq 0,0 \leq r(t) \leq r$, the following inequalities hold:

$$
\begin{aligned}
& -r \int_{t-r}^{t} \alpha^{T}(s) S \alpha(s) d s \\
& \quad \leq-\left[\begin{array}{l}
\int_{t-r(t)}^{t} \alpha(s) d s \\
\int_{t-r}^{t-r(t)} \alpha(s) d s
\end{array}\right]^{T}\left[\begin{array}{cc}
S & G \\
G^{T} & S
\end{array}\right]\left[\begin{array}{l}
\int_{t-r(t)}^{t} \alpha(s) d s \\
\int_{t-r}^{t-r(t)} \alpha(s) d s
\end{array}\right] .
\end{aligned}
$$

Lemma 4 (see [54]). For any constant matrix $R \in \mathbb{R}^{n \times n}, R=$ $R^{T}>0$, a scalar function $h:=h(t)>0$, and a vector-valued function $\dot{z}:[-h, 0] \rightarrow \mathbb{R}^{n}$ such that the following integrations are well:

$$
\begin{aligned}
& -h \int_{t-h}^{t} \dot{z}^{T}(s) R \dot{z}(s) d s \\
& \quad \leq\left[\begin{array}{c}
z(t) \\
z(t-h)
\end{array}\right]^{T}\left[\begin{array}{cc}
-R & R \\
R & -R
\end{array}\right]\left[\begin{array}{c}
z(t) \\
z(t-h)
\end{array}\right] .
\end{aligned}
$$

Lemma 5 (see [17]). For any positive semidefinite matrices,

$$
R=\left[\begin{array}{lll}
R_{11} & R_{12} & R_{13} \\
R_{12}^{T} & R_{22} & R_{23} \\
R_{13}^{T} & R_{23}^{T} & R_{33}
\end{array}\right] \geq 0,
$$

the integral inequality holds as follows:

$$
\begin{aligned}
& \int_{t-h}^{t} \dot{z}^{T}(s) R_{33} \dot{z}^{T}(s) d s \\
& \leq \int_{t-h}^{t}\left[\begin{array}{c}
z(t) \\
z(t-h) \\
\dot{z}(s)
\end{array}\right]^{T}\left[\begin{array}{lll}
R_{11} & R_{12} & R_{13} \\
R_{12}^{T} & R_{22} & R_{23} \\
R_{13}^{T} & R_{23}^{T} & 0
\end{array}\right]\left[\begin{array}{c}
z(t) \\
z(t-h) \\
\dot{z}(s)
\end{array}\right] d s .
\end{aligned}
$$

\section{Main Results}

In this section we will give sufficient conditions under which system (6) is globally exponentially stable.

Theorem 6. For given scalars $r>0, h>0$, and $1>$ $h_{D}$, the origin system (6) with the neuron activation function $f(z(t))$ satisfying condition (5) and the time-varying delay $h(t)$ satisfying (2) is globally exponentially stable with the exponential convergence rate index $k$ if there exist $P>0$, $Z_{i}>0(i=1,2), R_{i}>0(i=1,2,3,4)$, diagonal matrices $H_{1}=\operatorname{diag}\left\{h_{11}, h_{12}, \ldots, h_{1 n}\right\}>0, H_{2}=$ $\operatorname{diag}\left\{h_{21}, h_{22}, \ldots, h_{2 n}\right\}>0, H_{3}=\operatorname{diag}\left\{h_{31}, h_{32}, \ldots, h_{3 n}\right\}>0$, $H_{4}=\operatorname{diag}\left\{h_{41}, h_{42}, \ldots, h_{4 n}\right\}>0, R=\operatorname{diag}\left\{r_{1}, r_{2}, \ldots, r_{n}\right\}>0$, $G=\operatorname{diag}\left\{g_{1}, g_{2}, \ldots, g_{n}\right\}>0$, any matrices $T_{i}(i=1,2,3,4)$, and

$$
\begin{aligned}
& {\left[\begin{array}{cc}
S & M \\
M^{T} & S
\end{array}\right]>0,} \\
& X=\left[\begin{array}{lll}
X_{11} & X_{12} & X_{13} \\
X_{12}^{T} & X_{22} & X_{23} \\
X_{13}^{T} & X_{23}^{T} & X_{33}
\end{array}\right]>0, \\
& Y=\left[\begin{array}{lll}
Y_{11} & Y_{12} & Y_{13} \\
Y_{12}^{T} & Y_{22} & Y_{23} \\
Y_{13}^{T} & Y_{23}^{T} & Y_{33}
\end{array}\right]>0, \\
& R_{3}+\left(1-h_{D}\right) R_{4}-X_{33}>0, \\
& R_{3}-Y_{33}>0
\end{aligned}
$$

such that the following symmetric linear matrix inequality holds:

$$
\Psi=\left[\begin{array}{ccccccccc}
\Psi_{11} & \Psi_{12} & \Psi_{13} & 0 & \Psi_{15} & T_{2} A_{2}-A_{0} T_{4}^{T} & 0 & T_{2} A_{3} & 0 \\
* & \Psi_{22} & 0 & 0 & \Psi_{25} & T_{1} A_{2}-T_{4}^{T} & 0 & T_{1} A_{3} & 0 \\
* & * & \Psi_{33} & \Psi_{34} & 0 & H_{3} L_{2} & 0 & 0 & 0 \\
* & * & * & \Psi_{44} & 0 & 0 & H_{4} L_{2} & 0 & \\
* & * & * & * & \Psi_{55} & T_{3} A_{2}+A_{1}^{T} T_{4}^{T} & 0 & T_{3} A_{3} & 0 \\
* & * & * & * & * & \Psi_{66} & 0 & T_{4} A_{3} & 0 \\
* & * & * & * & * & * & \Psi_{77} & 0 & 0 \\
* & * & * & * & * & * & * & -e^{-2 k r} S & -e^{-2 k r} M \\
* & * & * & * & * & * & * & * & -e^{-2 k r} S
\end{array}\right] \leq 0
$$


where

$$
\begin{aligned}
& \Psi_{11}=2 k P+4 k\left(L^{+} G-L^{-} R\right)+R_{1}+R_{2} \\
& +e^{-2 k h}\left(h X_{11}+\overrightarrow{X_{13}}\right) \\
& -\frac{e^{-2 k h}}{h}\left(R_{3}+\left(1-h_{D}\right) R_{4}-X_{33}\right)-H_{2} L_{1} \\
& -\overrightarrow{T_{2} A_{0}} \\
& \Psi_{12}=P+L^{+} G-L^{-} R-A_{0} T_{1}^{T}-T_{2}, \\
& \Psi_{13}=e^{-2 k h}\left(h X_{12}-X_{13}+X_{23}^{T}\right) \\
& -\frac{e^{-2 k h}}{h}\left(R_{3}+\left(1-h_{D}\right) R_{4}-X_{33}\right), \\
& \Psi_{15}=2 k(R-G)+H_{2} L_{2}+T_{2} A_{1}-A_{0} T_{3}^{T}, \\
& \Psi_{22}=h R_{3}+h R_{4}-\overrightarrow{T_{1}} \text {, } \\
& \Psi_{25}=R-G+T_{1} A_{1}-T_{3}, \\
& \Psi_{33}=-\left(1-h_{D}\right) R_{2} \\
& -\frac{e^{-2 k h}}{h}\left(R_{3}+\left(1-h_{D}\right) R_{4}-X_{33}+R_{3}-Y_{33}\right) \\
& +e^{-2 k h}\left(h X_{22}-\overrightarrow{X_{23}}+h Y_{11}+\overrightarrow{Y_{13}}\right)-H_{3} L_{1}, \\
& \Psi_{34}=\frac{e^{-2 k h}}{h}\left(R_{3}-Y_{33}\right)+e^{-2 k h}\left(h Y_{12}-Y_{13}+Y_{23}^{T}\right) \text {, } \\
& \Psi_{44}=-R_{1}-\frac{e^{-2 k h}}{h}\left(R_{3}-Y_{33}\right)+e^{-2 k h}\left(h Y_{22}-\overrightarrow{Y_{23}}\right) \\
& -H_{4} L_{1} \\
& \Psi_{55}=Z_{1}+Z_{3}+r^{2} S-H_{1}-H_{2}+\overrightarrow{T_{3} A_{1}} \text {, } \\
& \Psi_{66}=-\left(1-h_{D}\right) e^{-2 k h} Z_{2}-H_{3}+\overrightarrow{T_{4} A_{2}} \text {, } \\
& \Psi_{77}=-e^{-2 k h} Z_{2}-H_{4} .
\end{aligned}
$$

Proof. Consider an augmentation of LKF for system (6) as follows:

$$
\begin{aligned}
V\left(z_{t}\right)= & V_{1}\left(z_{t}\right)+V_{2}\left(z_{t}\right)+V_{3}\left(z_{t}\right)+V_{4}\left(z_{t}\right)+V_{5}\left(z_{t}\right) \\
& +V_{6}\left(z_{t}\right)
\end{aligned}
$$

where

$$
\begin{aligned}
& V_{1}\left(z_{t}\right)=e^{2 k t} z^{T}(t) P z(t), \\
& V_{2}\left(z_{t}\right)=2 e^{2 k t}\left[\sum_{i=1}^{n} r_{i} \int_{0}^{z_{i}(t)}\left(f_{i}(s)-l_{i}^{-} s\right) d s\right. \\
& \left.\quad+\sum_{i=1}^{n} g_{i} \int_{0}^{z_{i}(t)}\left(l_{i}^{+} s-f_{i}(s)\right) d s\right],
\end{aligned}
$$

$$
\begin{aligned}
& V_{3}\left(z_{t}\right)=\int_{t-h}^{t} e^{2 k s} z^{T}(s) R_{1} z(s) d s \\
& +\int_{t-h(t)}^{t} e^{2 k s} z^{T}(s) R_{2} z(s) d s, \\
& V_{4}\left(z_{t}\right)=\int_{-h}^{0} \int_{t+\theta}^{t} e^{2 k s} \dot{z}^{T}(s) R_{3} \dot{z}(s) d s d \theta \\
& \quad+\int_{-h(t)}^{0} \int_{t+\theta}^{t} e^{2 k s} \dot{z}^{T}(s) R_{4} \dot{z}(s) d s d \theta, \\
& V_{5}\left(z_{t}\right)=\int_{t-h}^{t} e^{2 k s} f^{T}(z(s)) Z_{1} f(z(s)) d s \\
& \quad+\int_{t-h(t)}^{t} e^{2 k s} f^{T}(z(s)) Z_{2} f(z(s)) d s, \\
& V_{6}\left(z_{t}\right)=r \int_{-r}^{0} \int_{t+\theta}^{t} e^{2 k s} f^{T}(z(s)) S f(z(s)) d s d \theta .
\end{aligned}
$$

The time derivative of $V\left(z_{t}\right)$ along with the trajectory of system (6) is given as

$$
\begin{aligned}
\dot{V}\left(z_{t}\right)= & \dot{V}_{1}\left(z_{t}\right)+\dot{V}_{2}\left(z_{t}\right)+\dot{V}_{3}\left(z_{t}\right)+\dot{V}_{4}\left(z_{t}\right)+\dot{V}_{5}\left(z_{t}\right) \\
& +\dot{V}_{6}\left(z_{t}\right)
\end{aligned}
$$

where

$$
\begin{aligned}
& \dot{V}_{1}\left(z_{t}\right)=2 k e^{2 k t} z^{T}(t) P z(t)+2 e^{2 k t} z^{T}(t) P \dot{z}(t), \\
& \dot{V}_{2}\left(z_{t}\right)=4 k e^{2 k t}\left[\left(f^{T}(z(t))-z^{T}(t) L^{-}\right) R z(t)\right. \\
& \left.+\left(z^{T}(t) L^{+}-f^{T}(z(t))\right) G z(t)\right] \\
& +2 e^{2 k t}\left[f^{T}(z(t))(R-G)+z^{T}(t)\left(L^{+} G-L^{-} R\right)\right] \\
& \cdot \dot{z}(t), \\
& \dot{V}_{3}\left(z_{t}\right)=e^{2 k t} z^{T}(t)\left(R_{1}+R_{2}\right) z(t)-e^{2 k t} z^{T}(t-h) \\
& \cdot R_{1} z(t-h)-(1-\dot{h}(t)) e^{2 k t} z^{T}(t-h(t)) R_{2} z(t \\
& -h(t)) \leq e^{2 k t} z^{T}(t)\left(R_{1}+R_{2}\right) z(t)-e^{2 k t} z^{T}(t-h) \\
& \cdot R_{1} z(t-h)-\left(1-h_{D}\right) e^{2 k t} z^{T}(t-h(t)) R_{2} z(t \\
& -h(t)),
\end{aligned}
$$




$$
\begin{aligned}
& \dot{V}_{4}\left(z_{t}\right)=e^{2 k t} \dot{z}^{T}(t)\left(h R_{3}+h(t) R_{4}\right) \dot{z}(t) \\
& \cdot Y_{33} \dot{z}(s) d s \leq e^{2 k t} h \dot{z}^{T}(t)\left(R_{3}+R_{4}\right) \dot{z}(t) \\
& -\int_{t-h}^{t} e^{2 k s} \dot{z}^{T}(s) R_{3} \dot{z}(s) d s-(1-\dot{h}(t)) \\
& -e^{2 k(t-h)} \int_{t-h(t)}^{t} \dot{z}^{T}(s)\left(R_{3}+\left(1-h_{D}\right) R_{4}-X_{33}\right) \\
& \cdot \int_{t-h(t)}^{t} e^{2 k s} \dot{z}^{T}(s) R_{5} \dot{z}(s) d s \leq e^{2 k t} h \dot{z}^{T}(t)\left(R_{3}+R_{4}\right) \\
& \cdot \dot{z}(s) d s-e^{2 k(t-h)} \int_{t-h}^{t-h(t)} \dot{z}^{T}(s)\left(R_{3}-Y_{33}\right) \dot{z}(s) d s \\
& \cdot \dot{z}(t)-\int_{t-h(t)}^{t} e^{2 k s} \dot{z}^{T}(s)\left(R_{3}+\left(1-h_{D}\right) R_{4}-X_{33}\right) \\
& -e^{2 k(t-h)} \int_{t-h(t)}^{t} \dot{z}^{T}(s) X_{33} \dot{z}(s) d s \\
& \cdot \dot{z}(s) d s-\int_{t-h}^{t-h(t)} e^{2 k s} \dot{z}^{T}(s)\left(R_{3}-Y_{33}\right) \dot{z}(s) d s \\
& -e^{2 k(t-h)} \int_{t-h}^{t-h(t)} \dot{z}^{T}(s) Y_{33} \dot{z}(s) d s .
\end{aligned}
$$$$
-\int_{t-h(t)}^{t} e^{2 k s} \dot{z}^{T}(s) X_{33} \dot{z}(s) d s-\int_{t-h}^{t-h(t)} e^{2 k s} \dot{z}^{T}(s)
$$

Using Lemma 4, we can have

$$
\begin{aligned}
& -e^{2 k(t-h)} \int_{t-h(t)}^{t} \dot{z}^{T}(s)\left(R_{3}+\left(1-h_{D}\right) R_{4}-X_{33}\right) \dot{z}(s) d s \\
& \leq-\frac{e^{2 k(t-h)}}{h}\left[\begin{array}{c}
z(t) \\
z(t-h(t))
\end{array}\right]^{T}\left[\begin{array}{cc}
\left(R_{3}+\left(1-h_{D}\right) R_{4}-X_{33}\right) & -\left(R_{3}+\left(1-h_{D}\right) R_{4}-X_{33}\right) \\
-\left(R_{3}+\left(1-h_{D}\right) R_{4}-X_{33}\right) & \left(R_{3}+\left(1-h_{D}\right) R_{4}-X_{33}\right)
\end{array}\right]\left[\begin{array}{c}
z(t) \\
z(t-h(t))
\end{array}\right] \\
& =-\frac{e^{2 k(t-h)}}{h} z^{T}(t)\left(R_{3}+\left(1-h_{D}\right) R_{4}-X_{33}\right) z(t)+2 \frac{e^{2 k(t-h)}}{h} z^{T}(t)\left(R_{3}+\left(1-h_{D}\right) R_{4}-X_{33}\right) z(t-h(t)) \\
& -\frac{e^{2 k(t-h)}}{h} z^{T}(t-h(t))\left(R_{3}+\left(1-h_{D}\right) R_{4}-X_{33}\right) z(t-h(t)) \\
& -e^{2 k(t-h)} \int_{t-h}^{t-h(t)} \dot{z}^{T}(s)\left(R_{3}-Y_{33}\right) \dot{z}(s) d s \leq-\frac{e^{2 k(t-h)}}{h}\left[\begin{array}{c}
z(t-h(t)) \\
z(t-h)
\end{array}\right]^{T}\left[\begin{array}{cc}
\left(R_{3}-Y_{33}\right) & -\left(R_{3}-Y_{33}\right) \\
-\left(R_{3}-Y_{33}\right) & \left(R_{3}-Y_{33}\right)
\end{array}\right]\left[\begin{array}{c}
z(t-h(t)) \\
z(t-h)
\end{array}\right] \\
& =-\frac{e^{2 k(t-h)}}{h} z^{T}(t-h(t))\left(R_{3}-Y_{33}\right) z(t-h(t))+2 \frac{e^{2 k(t-h)}}{h} z^{T}(t-h(t))\left(R_{3}-Y_{33}\right) z(t-h) \\
& -\frac{e^{2 k(t-h)}}{h} z^{T}(t-h)\left(R_{3}-Y_{33}\right) z(t-h) .
\end{aligned}
$$

Using Lemma 5, we may get

$$
\begin{aligned}
& -e^{2 k(t-h)} \int_{t-h(t)}^{t} \dot{z}^{T}(s) X_{33} \dot{z}(s) d s \\
& \leq e^{2 k(t-h)} \int_{t-h(t)}^{t}\left[\begin{array}{c}
z(t) \\
z(t-h(t)) \\
\dot{z}(s)
\end{array}\right] \\
& \cdot\left[\begin{array}{ccc}
X_{11} & X_{12} & X_{13} \\
X_{12}^{T} & X_{22} & X_{23} \\
X_{13}^{T} & X_{23}^{T} & 0
\end{array}\right]\left[\begin{array}{c}
z(t) \\
z(t-h(t)) \\
\dot{z}(s)
\end{array}\right] d s \\
& =e^{2 k(t-h)} z^{T}(t)\left(h(t) X_{11}+X_{13}^{T}+X_{13}\right) z(t) \\
& +2 e^{2 k(t-h)} z^{T}(t)\left(h(t) X_{12}-X_{13}+X_{23}^{T}\right) z(t
\end{aligned}
$$

$$
\begin{gathered}
-h(t))+e^{2 k(t-h)} z^{T}(t-h(t))\left(h(t) X_{22}-X_{23}^{T}\right. \\
\left.-X_{23}\right) z(t-h(t)) \leq e^{2 k(t-h)} z^{T}(t)\left(h X_{11}+X_{13}^{T}\right. \\
\left.+X_{13}\right) z(t)+2 e^{2 k(t-h)} z^{T}(t)\left(h X_{12}-X_{13}+X_{23}^{T}\right) \\
\cdot z(t-h(t))+e^{2 k(t-h)} z^{T}(t-h(t))\left(h X_{22}-X_{23}^{T}\right. \\
\left.-X_{23}\right) z(t-h(t)), \\
-e^{2 k(t-h)} \int_{t-h}^{t-h(t)} \dot{z}^{T}(s) Y_{33} \dot{z}(s) d s \\
\leq e^{2 k(t-h)} \int_{t-h}^{t-h(t)}\left[\begin{array}{c}
z(t-h(t)) \\
z(t-h) \\
\dot{z}(s)
\end{array}\right]
\end{gathered}
$$




$$
\begin{aligned}
& \cdot\left[\begin{array}{ccc}
Y_{11} & Y_{12} & Y_{13} \\
Y_{12}^{T} & Y_{22} & Y_{23} \\
Y_{13}^{T} & Y_{23}^{T} & 0
\end{array}\right]\left[\begin{array}{c}
z(t-h(t)) \\
z(t-h) \\
\dot{z}(s)
\end{array}\right] d s=e^{2 k(t-h)} z^{T}(t \\
- & h(t))\left((h-h(t)) Y_{11}+Y_{13}^{T}+Y_{13}\right) z(t-h(t)) \\
+ & 2 e^{2 k(t-h)} z^{T}(t-h(t))\left((h-h(t)) Y_{12}-Y_{13}+Y_{23}^{T}\right) \\
& -z(t-h)+e^{2 k(t-h)} z^{T}(t-h)\left((h-h(t)) Y_{22}-Y_{23}^{T}\right. \\
& \left.-Y_{23}\right) z(t-h) \leq e^{2 k(t-h)} z^{T}(t-h(t))\left(h Y_{11}+Y_{13}^{T}\right. \\
+ & \left.Y_{13}\right) z(t-h(t))+2 e^{2 k(t-h)} z^{T}(t-h(t))\left(h Y_{12}\right. \\
- & \left.Y_{13}+Y_{23}^{T}\right) z(t-h)+e^{2 k(t-h)} z^{T}(t-h)\left(h Y_{22}\right. \\
- & \left.Y_{23}^{T}-Y_{23}\right) z(t-h), \\
\dot{V}_{5} & \left(z_{t}\right) \leq e^{2 k t} f^{T}(z(t))\left(Z_{1}+Z_{2}\right) f(z(t)) \\
- & e^{2 k(t-h)} f^{T}(z(t-h)) Z_{1} f(z(t-h))-\left(1-h_{D}\right) \\
\cdot & e^{2 k(t-h)} f^{T}(z(t-h(t))) Z_{2} f(z(t-h(t))) .
\end{aligned}
$$

By Lemma 3, we can obtain

$$
\begin{gathered}
\dot{V}_{6}\left(z_{t}\right) \leq r^{2} e^{2 k t} f^{T}(z(t)) S f(z(t)) \\
-r \int_{t-r}^{t} e^{2 k s} f^{T}(z(s)) S f(z(s)) d s \\
\leq r^{2} e^{2 k t} f^{T}(z(t)) S f(z(t)) \\
-e^{2 k(t-r)}\left[\begin{array}{l}
\int_{t-r(t)}^{t} f(z(s)) d s \\
\int_{t-r}^{t-r(t)} f(z(s)) d s
\end{array}\right]^{T} \\
\cdot\left[\begin{array}{cc}
S & M \\
M^{T} & S
\end{array}\right]\left[\begin{array}{l}
\int_{t-r(t)}^{t} f(z(s)) d s \\
\int_{t-r}^{t-r(t)} f(z(s)) d s
\end{array}\right] .
\end{gathered}
$$

From (5), for any $n \times n$ diagonal matrices $H_{i}>0(i=$ $1,2, \ldots, 4)$, the following inequality holds:

$$
\begin{aligned}
0 & \leq e^{2 k t}\left[z^{T}(t) L H_{1} L z(t)-f^{T}(z(t)) H_{1} f(z(t))\right] \\
& +e^{2 k t}\left[-z^{T}(t) H_{2} L_{1} z(t)+2 z^{T}(t) H_{2} L_{2} f(z(t))\right. \\
& \left.-f^{T}(z(t)) H_{2} f(z(t))\right] \\
& +e^{2 k t}\left[-z^{T}(t-h(t)) H_{3} L_{1} z(t-h(t))\right. \\
& +2 z^{T}(t-h(t)) H_{3} L_{2} f(z(t-h(t))) \\
& \left.-f^{T}(z(t-h(t))) H_{3} f(z(t-h(t)))\right] \\
& +e^{2 k t}\left[-z^{T}(t-h) H_{4} L_{1} z(t-h)\right. \\
& +2 z^{T}(t-h) H_{4} L_{2} f(z(t-h)) \\
& \left.-f^{T}(z(t-h)) H_{4} f(z(t-h))\right] .
\end{aligned}
$$

Furthermore, for arbitrary matrices $T_{1}, T_{2}, T_{3}$, and $T_{4}$ with appropriate dimensions, we have

$$
\begin{aligned}
0 & =2\left[\dot{z}^{T}(t) T_{1}+z^{T}(t) T_{2}+f^{T}(z(t)) T_{3}\right. \\
& \left.+f^{T}(z(t-h(t))) T_{4}\right] \cdot\left[-\dot{z}(t)-A_{0} z(t)\right. \\
& +A_{1} f(z(t))+A_{2} f(z(t-h(t))) \\
& \left.+A_{3} \int_{t-r(t)}^{t} f(z(s)) d s\right] .
\end{aligned}
$$

The combination of (19)-(24) gives

$$
V\left(x_{t}\right) \leq \xi^{T}(t) \Psi \xi(t)
$$

where $\xi(t)=\left[\begin{array}{llllll}z(t) & \dot{z}(t) & z(t-h(t)) & z(t-h) & f(z(t)) & f(z(t-\end{array}\right.$ $\left.h(t))) f(z(t-h)) \quad \int_{t-r(t)}^{t} f(z(s)) d s \int_{t-r}^{t-r(t)} f(z(s)) d s\right]$.

From (14), we know that $\dot{V}\left(z_{t}\right)<0$, which means the asymptotically stability of system (5). This completes the proof.

Furthermore, setting $d=\max \{h, r\}$, we can have

$$
\begin{aligned}
& V_{1}(z(0)) \leq \lambda_{\max }(P)\|z(s)\|^{2} \leq \lambda_{\max }(P) \\
& \quad \sup _{-d \leq s \leq 0}\|z(s)\|^{2}, \\
& V_{2}(z(0)) \leq 2\left[f(z(0))-L^{-} z(0)\right]^{T} R z(0) \\
& \quad+2\left[L^{+} z(0)-f(z(0))\right]^{T} G z(0) \\
& \quad \leq 2 \lambda_{\max }\left(L^{+}-L^{-}\right)\left(\lambda_{\max }(R)+\lambda_{\max }(G)\right) \\
& \quad \cdot \sup _{-d \leq s \leq 0}\|z(s)\|^{2}, \\
& V_{3}(z(0)) \leq h\left[\lambda_{\max }\left(R_{1}\right)+\lambda_{\max }\left(R_{2}\right)\right] \sup _{-d \leq s \leq 0}\|z(s)\|^{2}, \\
& V_{4}(z(0)) \leq \lambda_{\max }\left(R_{3}\right) \int_{-h}^{0} \int_{t+\theta}^{t} \dot{z}^{T}(s) \dot{z}(s) d s d \theta \\
& \quad+\lambda_{\max }\left(R_{4}\right) \int_{-h(t)}^{0} \int_{t+\theta}^{t} \dot{z}^{T}(s) \dot{z}(s) d s d \theta .
\end{aligned}
$$

It is easy to have

$$
\begin{aligned}
& \dot{z}^{T}(s) \dot{z}(s)=\left[-A_{0} z(s)+A_{1} f(z(s))\right. \\
& \left.+A_{2} f(z(s-h(s)))+A_{3} \int_{s-r(s)}^{s} f(z(\theta)) d \theta\right]^{T} \\
& \cdot\left[-A_{0} z(s)+A_{1} f(z(s))+A_{2} f(z(s-h(s)))\right. \\
& \left.+A_{3} \int_{s-r(s)}^{s} f(z(\theta)) d \theta\right]=z^{T}(s) A_{0}^{T} A_{0} z(s) \\
& +f^{T}(z(s)) A_{1}^{T} A_{1} f(z(s))+f^{T}(z(s-h(s))) \\
& \cdot A_{2}^{T} A_{2} f(z(s-h(s)))
\end{aligned}
$$




$$
\begin{aligned}
& +\int_{s-r(s)}^{s} f^{T}(z(\theta)) d \theta A_{3}^{T} A_{3} \int_{s-r(s)}^{s} f(z(\theta)) d \theta \\
& -2 z^{T}(s) A_{0}^{T} A_{1} f(z(s))-2 z^{T}(s) \\
& \cdot A_{0}^{T} A_{2} f(z(s-h(s)))-2 z^{T}(s) \\
& \cdot A_{0}^{T} A_{3} \int_{s-r(s)}^{s} f(z(\theta)) d \theta+2 f^{T}(z(s)) \\
& \cdot A_{1}^{T} A_{2} f(z(s-h(s)))+2 f^{T}(z(s)) \\
& \cdot A_{1}^{T} A_{3} \int_{s-r(s)}^{s} f(z(\theta)) d \theta+f^{T}(z(s-h(s))) \\
& \cdot A_{2}^{T} A_{3} \int_{s-r(s)}^{s} f(z(\theta)) d \theta .
\end{aligned}
$$

According to $2 a^{T} b \leq a^{T} X a+b^{T} X^{-} b$ with $X>0$,

$$
\begin{aligned}
& \dot{z}^{T}(s) \dot{z}(s) \leq 4\left[\lambda_{\max }\left(A_{0}^{T} A_{0}\right)\right. \\
& +\lambda_{\max }^{2}(L) \lambda_{\max }\left(A_{1}^{T} A_{1}\right)+\lambda_{\max }^{2}(L) \lambda_{\max }\left(A_{2}^{T} A_{2}\right) \\
& \left.+r^{2} \lambda_{\max }^{2}(L) \lambda_{\max }\left(A_{3}^{T} A_{3}\right)\right] \sup _{-d \leq s \leq 0}\|z(s)\|^{2}, \\
& V_{5}(z(0)) \leq h \lambda_{\max }^{2}(L)\left(\lambda_{\max }\left(Z_{1}\right)+\lambda_{\max }\left(Z_{2}\right)\right) \\
& \cdot \sup _{-d \leq s \leq 0}\|z(s)\|^{2}, \\
& V_{6}(z(0)) \leq r \lambda_{\max }(S) \\
& \cdot \int_{-r}^{0} \int_{t+\theta}^{t} f^{T}(z(s)) f(z(s)) d s d \theta \leq \frac{r^{3}}{2} \lambda_{\max }^{2}(L) \\
& \cdot \lambda_{\max }(S) \sup _{-d \leq s \leq 0}\|z(s)\|^{2} .
\end{aligned}
$$

Thus according to (26)-(28), there exists a positive constant $\gamma$ such that

$$
V(z(0))<\gamma \sup _{-d \leq s \leq 0}\|z(s)\|^{2}
$$

where

$$
\begin{aligned}
\gamma & =\lambda_{\max }(P)+2 \lambda_{\max }\left(L^{+}-L^{-}\right)\left(\lambda_{\max }(R)\right. \\
& \left.+\lambda_{\max }(G)\right)+h\left[\lambda_{\max }\left(R_{1}\right)+\lambda_{\max }\left(R_{2}\right)\right] \\
& +2 h^{2}\left[\left(\lambda_{\max }\left(R_{3}\right)+\lambda_{\max }\left(R_{4}\right)\right)\right]\left[\lambda_{\max }\left(A_{0}^{T} A_{0}\right)\right. \\
& +\lambda_{\max }^{2}(L) \lambda_{\max }\left(A_{1}^{T} A_{1}\right)+\lambda_{\max }^{2}(L) \lambda_{\max }\left(A_{2}^{T} A_{2}\right) \\
& \left.+r^{2} \lambda_{\max }^{2}(L) \lambda_{\max }\left(A_{3}^{T} A_{3}\right)\right]+h \lambda_{\max }^{2}(L) \\
& \cdot\left(\lambda_{\max }\left(Z_{1}\right)+\lambda_{\max }\left(Z_{2}\right)\right)+\frac{r^{3}}{2} \\
& \cdot \lambda_{\max }^{2}(L) \lambda_{\max }(S) .
\end{aligned}
$$

On the other hand, we have

$$
V(z(t)) \geq e^{2 k t} \lambda_{\min }(P)\|z(t)\|^{2} .
$$

Therefore

$$
\|z(t)\| \geq \sqrt{\frac{\gamma}{\lambda_{\min }(P)}} e^{-k t} \sup _{-d \leq s \leq 0}\|z(s)\| .
$$

Then, from Definition 1, system (6) is exponentially stable with convergence rate $k$, and the proof is completed.

Remark 7. In the paper, the reduced conservatism of Theorem 6 benefits primarily from a new integral inequality, which is proved to be less conservative than the celebrated Jensen's inequality, and takes fully the relationship between the terms in the Leibniz-Newton formula within the framework of LMIs into account. In order to lower the conservatism of stability criteria, we further deal with the integral terms of $e^{2 k(t-h)} \int_{t-h(t)}^{t} \dot{z}^{T}(s)\left(R_{3}+\left(1-h_{D}\right) R_{4}-X_{33}\right) \dot{z}(s) d s$ and $e^{2 k(t-h)} \int_{t-h}^{t-h(t)} \dot{z}^{T}(s)\left(R_{3}-Y_{33}\right) \dot{z}(s) d s$ via Lemma 4. Different from that of [17], this kind of processing method can reduce ulteriorly the conservatism of stability criteria.

Remark 8. As a matter of fact, Theorem 6 gives a stability criterion for system (6) with $h(t)$ satisfying $0 \leq h(t) \leq h$, $0 \leq \dot{h}(t) \leq h_{D}$, where $h_{D}$ is given constant. In many cases, $h_{D}$ is unknown. Considering this situation, a rate-independent corollary for the delay $h(t)$ satisfying $0 \leq h(t) \leq h$ is derived by setting $R_{2}=0, R_{4}=0$, and $Z_{2}=0$ in the proof of Theorem 6 .

Theorem 9. For given scalars $0<r$ and $h>0$, the origin of system (6) with the neuron activation function $f(z(t))$ satisfying condition (5) is globally exponentially stable with the exponential convergence rate index $k$ if there exist $P>0$, $Z_{1}>0, R_{i}^{T}=R_{i}>0(i=1,3)$, diagonal matrices $H_{1}=$ $\operatorname{diag}\left\{h_{11}, h_{12}, \ldots, h_{1 n}\right\}>0, H_{2}=\operatorname{diag}\left\{h_{21}, h_{22}, \ldots, h_{2 n}\right\}>0$, $H_{3}=\operatorname{diag}\left\{h_{31}, h_{32}, \ldots, r_{3 n}\right\}>0, H_{4}=\operatorname{diag}\left\{h_{41}, h_{42}, \ldots\right.$, $\left.h_{4 n}\right\}>0, R=\operatorname{diag}\left\{r_{1}, r_{2}, \ldots, r_{n}\right\}>0, G=\operatorname{diag}\left\{g_{1}, g_{2}, \ldots\right.$, $\left.g_{n}\right\}>0$, any matrices $T_{i}(i=1,2, \ldots, 4)$, and

$$
\begin{array}{rl}
{\left[\begin{array}{cc}
S & M \\
M^{T} & S
\end{array}\right]>} & 0, \\
X & =\left[\begin{array}{lll}
X_{11} & X_{12} & X_{13} \\
X_{12}^{T} & X_{22} & X_{23} \\
X_{13}^{T} & X_{23}^{T} & X_{33}
\end{array}\right]>0, \\
Y & =\left[\begin{array}{lll}
Y_{11} & Y_{12} & Y_{13} \\
Y_{12}^{T} & Y_{22} & Y_{23} \\
Y_{13}^{T} & Y_{23}^{T} & Y_{33}
\end{array}\right]>0, \\
R_{3}-X_{33}>0 & 0, \\
R_{3}-Y_{33}>0 &
\end{array}
$$


such that the following symmetric linear matrix inequality holds:

$$
\Phi=\left[\begin{array}{ccccccccc}
\Phi_{11} & \Phi_{12} & \Phi_{13} & 0 & \Phi_{15} & T_{2} A_{2}-A_{0} T_{4}^{T} & 0 & T_{2} A_{3} & 0 \\
* & \Phi_{22} & 0 & 0 & \Phi_{25} & T_{1} A_{2}-T_{4}^{T} & 0 & T_{1} A_{3} & 0 \\
* & * & \Phi_{33} & \Phi_{34} & 0 & H_{3} L_{2} & 0 & 0 & 0 \\
* & * & * & \Phi_{44} & 0 & 0 & H_{4} L_{2} & 0 & \\
* & * & * & * & \Phi_{55} & T_{3} A_{2}+A_{1}^{T} T_{4}^{T} & 0 & T_{3} A_{3} & 0 \\
* & * & * & * & * & \Phi_{66} & 0 & T_{4} A_{3} & 0 \\
* & * & * & * & * & * & \Phi_{77} & 0 & 0 \\
* & * & * & * & * & * & * & -e^{-2 k r} S & -e^{-2 k r} M \\
* & * & * & * & * & * & * & * & -e^{-2 k r} S
\end{array}\right] \leq 0,
$$

where

$$
\begin{aligned}
& \Phi_{11}=2 k P+4 k\left(L^{+} G-L^{-} R\right)+R_{1} \\
& +e^{-2 k h}\left(h X_{11}+\overrightarrow{X_{13}}\right)-\frac{e^{-2 k h}}{h}\left(R_{3}-X_{33}\right) \\
& -H_{2} L_{1}-\overrightarrow{T_{2} A_{0}} \\
& \Phi_{12}=P+L^{+} G-L^{-} R-A_{0} T_{1}^{T}-T_{2} \text {, } \\
& \Phi_{13}=e^{-2 k h}\left(h X_{12}-X_{13}+X_{23}^{T}\right)-\frac{e^{-2 k h}}{h}\left(R_{3}-X_{33}\right) \text {, } \\
& \Phi_{15}=2 k(R-G)+H_{2} L_{2}+T_{2} A_{1}-A_{0} T_{3}^{T} \text {, } \\
& \Phi_{22}=h R_{3}-\vec{T}_{1} \text {, } \\
& \Phi_{25}=R-G+T_{1} A_{1}-T_{3} \text {, } \\
& \Phi_{33}=-\left(1-h_{D}\right) R_{2}-\frac{e^{-2 k h}}{h}\left(2 R_{3}-X_{33}-Y_{33}\right) \\
& +e^{-2 \mathrm{k} h}\left(h X_{22}-\overrightarrow{X_{23}}+h Y_{11}+\overrightarrow{Y_{13}}\right) \\
& -H_{3} L_{1} \\
& \Phi_{34}=\frac{e^{-2 k h}}{h}\left(R_{3}-Y_{33}\right)+e^{-2 k h}\left(h Y_{12}-Y_{13}+Y_{23}^{T}\right) \text {, } \\
& \Phi_{44}=-R_{1}-\frac{e^{-2 k h}}{h}\left(R_{3}-Y_{33}\right)+e^{-2 k h}\left(h Y_{22}-\overrightarrow{Y_{23}}\right) \\
& -H_{4} L_{1} \text {, } \\
& \Phi_{55}=Z_{1}+r^{2} S-H_{1}-H_{2}+\overrightarrow{T_{3} A_{1}} \text {, } \\
& \Phi_{66}=-H_{3}+\overrightarrow{T_{4} A_{2}} \text {, } \\
& \Phi_{77}=-e^{-2 k h} Z_{2}-H_{4} \text {. }
\end{aligned}
$$

The other procedure is straight forward from the proof of Theorem 6, so we omit it.

Remark 10. In the paper, we make full use of the relationship between $\int_{t-r(t)}^{t} f(z(s)) d s$ and $\int_{t-r}^{t-r(t)} f(z(s)) d s$, which can reduce the conservatism of stability criteria once again. However, these useful terms of $\quad \int_{t-r(t)}^{t} f^{T}(z(s)) d s M \int_{t-r}^{t-r(t)} f(z(s)) d s \quad$ and $\int_{t-\mathrm{r}}^{t-r(t)} f^{T}(z(s)) d s S \int_{t-r}^{t-r(t)} f(z(s)) d s$ were always ignored in [18-20], which may lead to considerable conservatism to certain extent.

Remark 11. Due to constructing a simple type of LyapunovKrasovskii functional and taking full advantage of effective mathematical techniques, the conservatism of improved delay-dependent stability criteria obtained is reduced to a great degree in this study. Compared with those in previous articles [22, 23], we employ a few free variables and do not use a delay decomposition method and add some zero terms, that is, not only dividing the discrete delay interval $[0, d]$ into two ones $[0, d / 2]$ and $[d / 2, d]$, but also dividing the discrete delay interval $[0, d]$ into three ones $[0, d(t) / 2],[d(t) / 2, d(t)]$, and $[d(t), d]$ and adding the following equalities:

$$
\begin{aligned}
& 2 e^{2 k(t-d / 2)} \zeta_{1}^{T}(t) U\left[z\left(t-\frac{d(t)}{2}\right)-z\left(t-\frac{d}{2}\right)\right. \\
& \left.-\int_{t-d / 2}^{t-d(t) / 2} \dot{z}(s) d s\right]=0, \\
& 2 e^{2 k(t-d / 2)} \zeta_{1}^{T}(t) V\left[z\left(t-\frac{d}{4}\right)-z\left(t-\frac{d(t)}{2}\right)\right. \\
& \left.-\int_{t-d / 2}^{t-d / 4} \dot{z}(s) d s\right]=0, \\
& 2 e^{2 k(t-d / 2)} \zeta_{1}^{T}(t) H\left[z(t)-z\left(t-\frac{d}{4}\right)\right.
\end{aligned}
$$




$$
\begin{aligned}
& \left.-\int_{t-d / 4}^{t} \dot{z}(s) d s\right]=0 \\
& 2 e^{2 k(t-d)} \zeta_{1}^{T}(t) W[z(t-d(t))-z(t-d) \\
& \left.-\int_{t-d}^{t-d(t)} \dot{z}(s) d s\right]=0 \\
& 2 e^{2 k(t-d)} \zeta_{1}^{T}(t) M\left[z\left(t-\frac{d}{2}\right)-z(t-d(t))\right. \\
& \left.-\int_{t-d(t)}^{t-d / 2} \dot{z}(s) d s\right]=0 \\
& 2 e^{2 k(t-d)}\left[\frac{d}{2} \zeta_{1}^{T}(t) Z \zeta_{1}(t)-\int_{t-d}^{t-d(t)} \zeta_{1}^{T}(t) Z \zeta_{1}(t) d s\right. \\
& \left.-\int_{t-d(t)}^{t-d / 2} \zeta_{1}^{T}(t) Z \zeta_{1}(t) d s\right]=0 \\
& 2 e^{2 k(t-d / 2)}\left[\frac{d}{2} \zeta_{1}^{T}(t) Y \zeta_{1}(t)-\int_{t-d / 2}^{t-d(t) / 2} \zeta_{1}^{T}(t) Y \zeta_{1}(t) d s\right. \\
& -\int_{t-d(t) / 2}^{t-d / 4} \zeta_{1}^{T}(t) Y \zeta_{1}(t) d s \\
& \left.-\int_{t-d / 4}^{t} \zeta_{1}^{T}(t) Y \zeta_{1}(t) d s\right]=0
\end{aligned}
$$

By using this method, the conservatism of the obtained stability condition in $[22,23]$ is reduced to some degree. However, the computing complexity is also improved since more variables are involved. Besides, we provided a comparison of the numbers of the variables involved in $[22,23]$ and our paper in Table 1. From Table 1, it is clear to see that the number of decision variables in our paper is much less than those in $[22,23]$. Thus, it also expounds validity and applicability of the proposed method.

Remark 12. In many actual applications, maximum allowable time-delay upper bounds $h$ are of interest. In Theorems 6 and 9 , with a fixed $h_{D}$ and $r, k$ can be obtained through following optimization procedure:

$$
\begin{aligned}
& \text { Maximize } h, \\
& \text { Subject to (14) or (34). }
\end{aligned}
$$

Besides, maximum allowable time-delay upper bounds $k$ obtained are very valuable. In Theorems 6 and 9, with a fixed $h_{D}$ and $r, h$ can be also acquired through following optimization procedure:

$$
\begin{array}{ll}
\text { Maximize } & k, \\
\text { Subject to } & (14) \text { or }(34) \text {. }
\end{array}
$$

Inequalities (37) and (38) are a convex optimization problem and can be obtained efficiently by using the MATLABLMI Toolbox.
TABLE 1: Comparison of the numbers of the involved variables.

\begin{tabular}{lccc}
\hline $\begin{array}{l}\text { Method/number of } \\
\text { decision variables }\end{array}$ & {$[22]$} & {$[23]$} & This paper \\
\hline Theorem 6 & $41 n^{2}+14 n$ & $52 n^{2}+18 n$ & $14 n^{2}+13 n$ \\
\hline Theorem 9 & $\frac{79}{2} n^{2}+\frac{25}{2} n$ & $\frac{101}{2} n^{2}+\frac{33}{2} n$ & $\frac{25}{2} n^{2}+\frac{23}{2} n$ \\
\hline
\end{tabular}

TABLE 2: Allowable upper bounds of $k$ for $k$ for Case A in Example 1.

\begin{tabular}{lcccccc}
\hline Method & {$[20]$} & {$[21]$} & {$[24]$} & {$[23]$} & {$[22]$} & Theorem 6 \\
\hline $\begin{array}{l}h=0.5, r=0.2, \\
h_{D}=0\end{array}$ & 0.46 & 0.58 & 0.56 & 0.67 & 0.80 & 0.88 \\
\hline $\begin{array}{l}h=0.5, r=0.2, \\
h_{D}=0.5\end{array}$ & 0.21 & 0.35 & 0.35 & 0.45 & 0.50 & 0.65 \\
$\begin{array}{l}h=0.6, r=0.2, \\
h_{D}=0.5\end{array}$ & 0.06 & 0.20 & 0.33 & 0.29 & 0.34 & 0.45 \\
$\begin{array}{l}h=0.8, r=0.2, \\
h_{D}=0.5\end{array}$ & 0.00 & 0.05 & 0.10 & 0.11 & 0.14 & 0.21 \\
\hline
\end{tabular}

\section{Numerical Examples}

In this section, three examples are given to demonstrate the feasibility and effectiveness of the main results derived above.

Example 1. Consider a delayed neural network in (6) with parameters as follows:

$$
\begin{aligned}
& A_{0}=\left[\begin{array}{lll}
6 & 0 & 0 \\
0 & 5 & 0 \\
0 & 0 & 7
\end{array}\right], \\
& A_{1}=\left[\begin{array}{ccc}
1.2 & -0.8 & 0.6 \\
0.5 & -1.5 & 0.7 \\
-0.8 & -1.2 & -1.4
\end{array}\right], \\
& A_{2}=\left[\begin{array}{ccc}
-1.4 & 0.9 & 0.5 \\
-0.6 & 1.2 & 0.8 \\
0.5 & -0.7 & 1.1
\end{array}\right], \\
& A_{3}=\left[\begin{array}{ccc}
1.8 & 0.7 & -0.8 \\
1.8 & 0.7 & -0.8 \\
-0.4 & -0.6 & 1.2
\end{array}\right] .
\end{aligned}
$$

Case $A$ (set $\left.L^{-}=\operatorname{diag}\{-1.2,0,-2.4\}, L^{+}=\operatorname{diag}\{0,1.4,0\}\right)$. For different $h$ and $h_{D}$, the allowable upper bounds of the exponential convergence rate index $k$ calculated by Theorem 6 in this paper and Theorem 1 in [20-24] are listed in Table 2. According to Table 2, this example is given to indicate significant improvements over some existing results.

Besides, for the parameters listed above, let $r=0.2, h=$ $0.5, h_{D}=0.5$, and $k=0.65$. Then we can obtain the following feasible parameters by Theorem 6 in our paper. Due to 
the limitation of the length of this paper, we only provide a part of the feasible solutions here as follows:

$$
\begin{aligned}
& P=\left[\begin{array}{ccc}
18.3158 & -9.1756 & 8.2601 \\
-9.1756 & 15.4632 & -3.5161 \\
8.2601 & -3.5161 & 12.6661
\end{array}\right] \\
& Z_{1}=\left[\begin{array}{ccc}
11.6082 & 0.1957 & -0.2876 \\
0.1957 & 11.0661 & -0.9006 \\
-0.2876 & -0.9006 & 11.3939
\end{array}\right] \text {, } \\
& Z_{2}=\left[\begin{array}{rrr}
19.9317 & -6.4931 & -3.0095 \\
-6.4931 & 21.7679 & -1.9799 \\
-3.0095 & -1.9799 & 30.9316
\end{array}\right] \text {, } \\
& R_{1}=\left[\begin{array}{ccc}
14.8956 & -0.4754 & 1.0203 \\
-0.4754 & 14.9458 & 0.3653 \\
1.0203 & 0.3653 & 17.8456
\end{array}\right] \text {, } \\
& R_{2}=\left[\begin{array}{ccc}
20.5413 & 2.7925 & 0.1017 \\
2.7925 & 21.4962 & 1.6793 \\
0.1017 & 1.6793 & 25.1932
\end{array}\right] \text {, } \\
& R_{3}=\left[\begin{array}{ccc}
5.6240 & -3.0814 & 2.1045 \\
-3.0814 & 5.4295 & -1.2819 \\
2.1045 & -1.2819 & 5.1175
\end{array}\right] \text {, } \\
& R_{4}=\left[\begin{array}{ccc}
1.9845 & -1.3207 & 0.9292 \\
-1.3207 & 1.8417 & -0.6156 \\
0.9292 & -0.6156 & 1.6339
\end{array}\right] \text {, } \\
& T_{1}=\left[\begin{array}{ccc}
4.0278 & -1.9174 & 0.9670 \\
-1.6145 & 4.2443 & -0.2659 \\
1.1456 & -1.1396 & 3.9531
\end{array}\right] \text {, } \\
& T_{2}=\left[\begin{array}{ccc}
9.0432 & -0.7086 & 0.7264 \\
-0.8280 & 11.0177 & 0.5688 \\
0.5356 & -0.9775 & 10.0672
\end{array}\right] \text {, } \\
& T_{3}=\left[\begin{array}{ccc}
-2.0541 & 0.5035 & -1.2211 \\
-1.2754 & 2.2408 & -1.7175 \\
1.0163 & 1.5246 & -3.3814
\end{array}\right] \text {, } \\
& X_{11}=\left[\begin{array}{ccc}
13.8117 & -0.5993 & 0.7694 \\
-0.5993 & 13.5660 & 0.1550 \\
0.7694 & 0.1550 & 14.4814
\end{array}\right] \text {, } \\
& X_{12}=\left[\begin{array}{ccc}
-1.2696 & 0.2376 & 0.0313 \\
0.2855 & -1.2632 & 0.2238 \\
-0.1326 & 0.1963 & -0.6930
\end{array}\right] \text {, }
\end{aligned}
$$$$
X_{13}=\left[\begin{array}{ccc}
0.1994 & -0.4629 & 0.4020 \\
-0.3868 & -0.0134 & -0.1262 \\
0.4274 & -0.2264 & 0.0949
\end{array}\right] \text {, }
$$$$
X_{22}=\left[\begin{array}{ccc}
11.6577 & -0.9613 & 0.8217 \\
-0.9613 & 11.2995 & -0.0487 \\
0.8217 & -0.0487 & 11.7214
\end{array}\right] \text {, }
$$$$
X_{23}=\left[\begin{array}{ccc}
0.6172 & 0.1352 & -0.1692 \\
-0.0092 & 0.8882 & 0.0132 \\
0.0119 & 0.0008 & 0.9916
\end{array}\right] \text {, }
$$$$
X_{33}=\left[\begin{array}{ccc}
3.3075 & -1.9174 & 1.3511 \\
-1.9174 & 3.0607 & -0.8479 \\
1.3511 & -0.8479 & 2.8448
\end{array}\right] \text {, }
$$$$
Y_{11}=\left[\begin{array}{ccc}
11.6312 & -0.9820 & 0.8535 \\
-0.9820 & 11.2608 & -0.0736 \\
0.8535 & -0.0736 & 11.6236
\end{array}\right] \text {, }
$$$$
Y_{12}=\left[\begin{array}{ccc}
-1.3825 & 0.2516 & -0.0664 \\
0.2417 & -1.5193 & 0.1776 \\
-0.0436 & 0.1741 & -1.3352
\end{array}\right] \text {, }
$$$$
Y_{13}=\left[\begin{array}{ccc}
-0.5087 & -0.1303 & 0.1473 \\
-0.0614 & -0.6698 & -0.0360 \\
0.1100 & -0.0658 & -0.6497
\end{array}\right] \text {, }
$$$$
Y_{22}=\left[\begin{array}{ccc}
13.8730 & -0.4984 & 0.5534 \\
-0.4984 & 13.6908 & 0.0897 \\
0.5534 & 0.0897 & 14.0093
\end{array}\right] \text {, }
$$$$
Y_{23}=\left[\begin{array}{ccc}
-0.1907 & 0.3597 & -0.2902 \\
0.3244 & -0.1140 & 0.1213 \\
-0.2874 & 0.1574 & -0.1037
\end{array}\right] \text {, }
$$$$
Y_{33}=\left[\begin{array}{ccc}
2.8308 & -1.5833 & 1.1085 \\
-1.5833 & 2.6778 & -0.6906 \\
1.1085 & -0.6906 & 2.5054
\end{array}\right] \text {. }
$$

Case $B$ (set $\left.L^{-}=\operatorname{diag}\{0,0,0\}, L^{+}=\operatorname{diag}\{0.5,0.5,0.5\}\right)$. When $k=0.5$ and $r=1$, the maximum allowable upper bounds $h$ obtained from Theorem 6 for different values $h_{D}$ are listed in Table 3. From Table 3, it can be seen clearly that our results have less conservatism over the results acquired by $[20,22,34-36]$ and illustrate the effectiveness and the advantage of the proposed methods. The active functions are chosen as $f_{1}(z(s))=0.25(|z(s)+1|-|z(s)-1|), f_{2}(z(s))=$ $0.25(|z(s)+1|-|z(s)-1|)$, and $f_{3}(z(s))=0.25(|z(s)+1|-$ $|z(s)-1|)$. Moreover, the globally exponentially stable with the initial value $[-2.5,-0.5,2.5]$ is shown in Figures $1-3$. 
TABLE 3: Maximum allowable time delay upper bounds $h$ for Case B in Example 1.

\begin{tabular}{lcccccc}
\hline Method & {$[20]$} & {$[34]$} & {$[35]$} & {$[36]$} & {$[22]$} & Theorem 6 \\
\hline$h_{D}=0$ & 2.2931 & 3.3294 & 5.8415 & 7.2820 & 9.6503 & 9.8356 \\
\hline$h_{D}=0.5$ & 2.1010 & 2.6363 & 4.0130 & 5.8958 & 8.0394 & 8.2175 \\
\hline$h_{D}=0.8$ & 1.7939 & 1.7200 & 3.6574 & 4.0847 & 5.8974 & 6.0561 \\
\hline
\end{tabular}

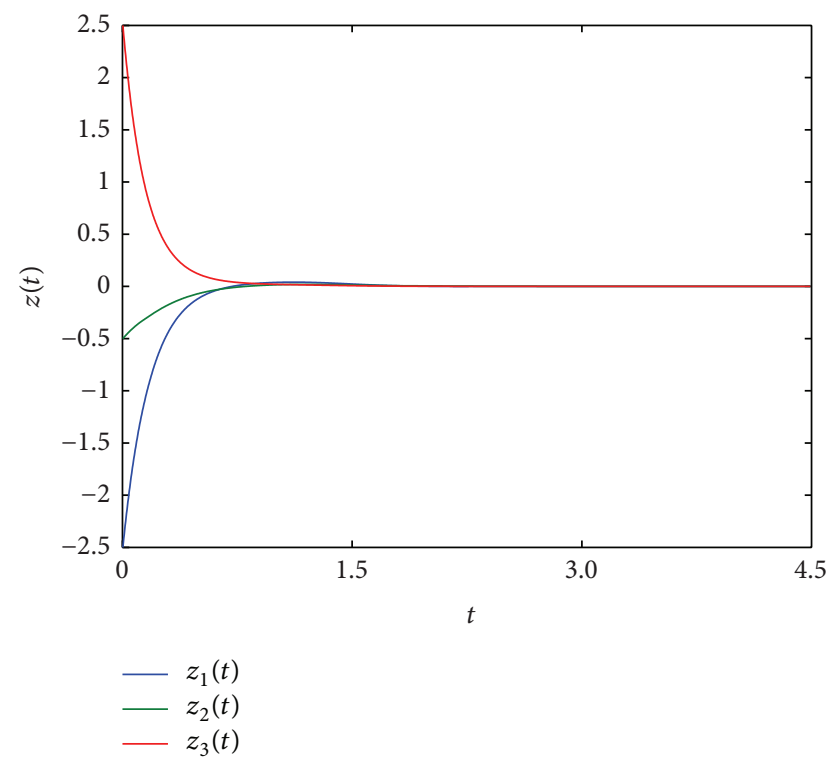

FIGURE 1: State trajectories of $z(t)$ in the plane for $h=9.8356$ in Example 1.

Example 2. Consider a delayed neural network in (6) with parameters as follows:

$$
\begin{aligned}
& A_{0}=\left[\begin{array}{ccc}
2.3 & 0 & 0 \\
0 & 3.4 & 0 \\
0 & 0 & 2.5
\end{array}\right], \\
& A_{1}=\left[\begin{array}{ccc}
0.9 & -1.5 & 0.1 \\
-1.2 & 1 & 0.2 \\
0.2 & 0.3 & 0.8
\end{array}\right], \\
& A_{2}=\left[\begin{array}{lll}
0.8 & 0.6 & 0.2 \\
0.5 & 0.7 & 0.1 \\
0.2 & 0.1 & 0.5
\end{array}\right], \\
& A_{3}=\left[\begin{array}{lll}
0.3 & 0.2 & 0.1 \\
0.1 & 0.2 & 0.1 \\
0.1 & 0.1 & 0.2
\end{array}\right] .
\end{aligned}
$$

Let $L^{-}=\operatorname{diag}\{0,0,0\}$ and let $L^{+}=\operatorname{diag}\{0.2,0.2,0.2\}$.

Case A. For $h=r$ the corresponding upper bounds of $h$ for unknown $h_{D}$ obtained by Theorem 9 and the results in [1820] are listed in Table 4. According to Table 4, this example
TABLE 4: Maximum allowable time delay upper bounds $h=r$ in Example 2.

\begin{tabular}{lcccc}
\hline Method & {$[19]$} & {$[18]$} & {$[20]$} & Theorem 9 \\
\hline$h=r$ & 1.833 & 3.597 & 5.068 & 5.220 \\
\hline
\end{tabular}

TABLE 5: Maximum allowable time delay upper bounds $r$ for different values $k$ in Example 2 .

\begin{tabular}{lcccc}
\hline Method & 0.1 & 0.3 & 0.5 & 0.8 \\
\hline$[22]$ & 9.082 & 4.774 & 3.313 & 2.217 \\
\hline Theorem 9 & 9.235 & 4.963 & 3.518 & 2.363 \\
\hline
\end{tabular}

TABLE 6: Comparison of the maximum delay $h$ between different methods for various $h_{D}$ in Example 3 .

\begin{tabular}{lcccc}
\hline$h_{D}$ & 0 & 0.2 & 0.5 & 0.7 \\
\hline$[24]$ & 3.377 & 3.336 & 3.268 & 3.264 \\
\hline Theorem 6 & 3.518 & 3.427 & 3.358 & 3.386 \\
\hline
\end{tabular}

shows that the stability criterion in this paper gives much less conservative results than those in the previous literatures.

The active functions are set as $f_{1}(z(s))=0.1(|z(s)+1|-$ $|z(s)-1|), f_{2}(z(s))=0.1(|z(s)+1|-|z(s)-1|)$, and $f_{3}(z(s))=$ $0.1(|z(s)+1|-|z(s)-1|)$; the globally exponentially stable with the initial value $[-0.4,-0.2,0.5]$ is shown in Figures $4-6$.

Case B. For $h=1$, the corresponding upper bounds of $r$ for unknown $h_{D}$ obtained by Theorem 9 are listed in Table 5. From Table 5, it is shown clearly that our results have significant improvement over the existing results.

Example 3. Consider a delayed neural network in (6) with parameters as follows:

$$
\begin{aligned}
& A_{0}=\left[\begin{array}{cc}
3.99 & 0 \\
0 & 2.99
\end{array}\right], \\
& A_{1}=\left[\begin{array}{cc}
1.188 & 0.09 \\
0.09 & 1.188
\end{array}\right], \\
& A_{2}=\left[\begin{array}{ll}
0.009 & 0.14 \\
0.05 & 0.09
\end{array}\right], \\
& A_{3}=\left[\begin{array}{ll}
0.45 & -0.2 \\
0.3 & 0.42
\end{array}\right] .
\end{aligned}
$$

Let $L^{-}=\operatorname{diag}\{0,0\}$ and let $L^{+}=\operatorname{diag}\{1,1\}$.

When $k=0.5$ and $r=0.1$, the corresponding upper bounds of $h$ for different $h_{D}$ obtained by Theorem 6 are listed in Table 6. The active functions are chosen as $f_{1}(z(s))=$ $0.5(|z(s)+1|-|z(s)-1|)$ and $f_{2}(z(s))=0.5(|z(s)+1|-$ $|z(s)-1|)$; the globally exponentially stable with the initial value $[-0.6,0.8]$ is given in Figures $7-9$. Therefore, we can say that the results in this paper are less conservative than those in [24]. 

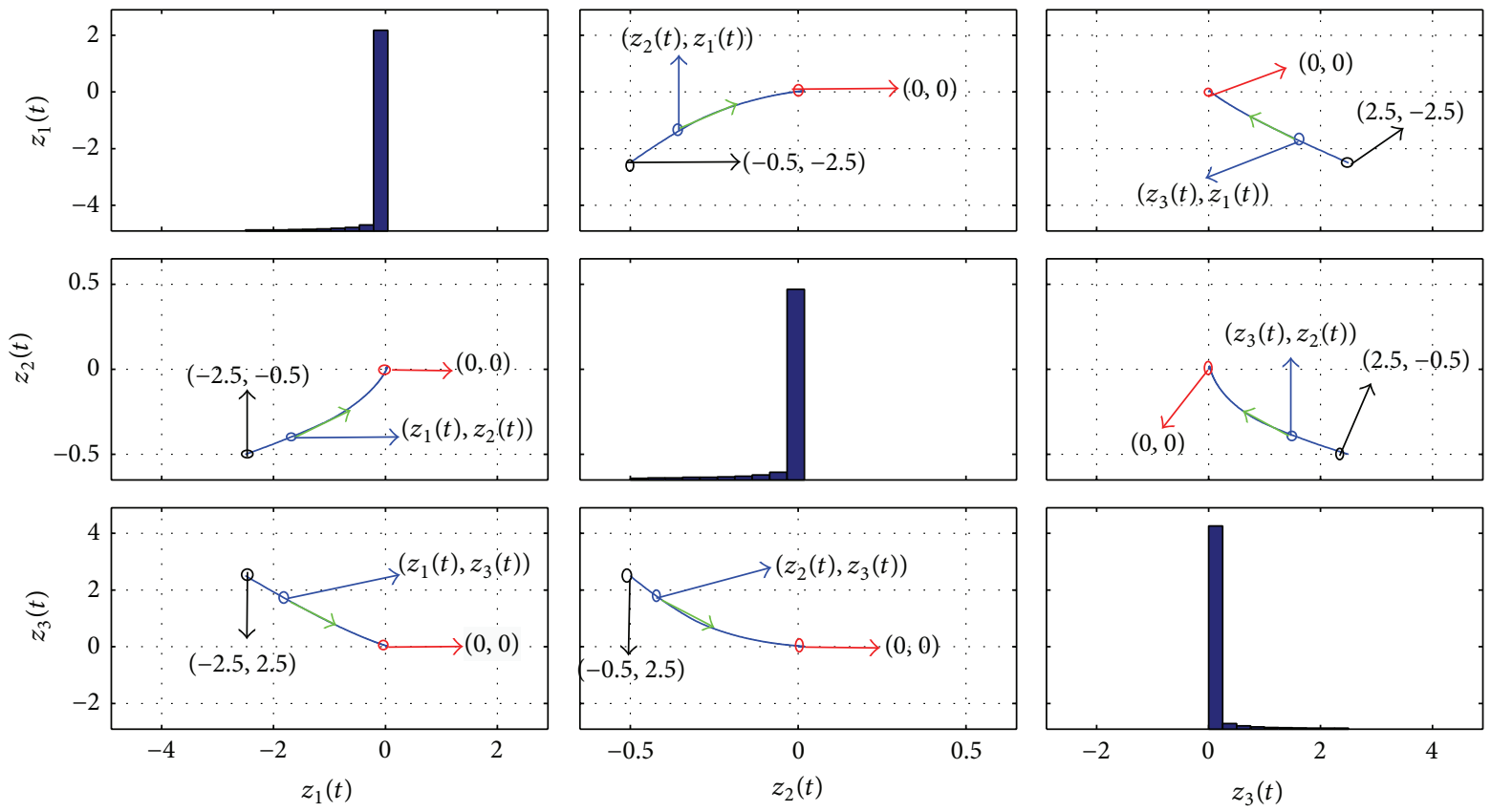

FIGURE 2: Phase trajectory of model of $z(t)$ in the plane for $h=9.8356$ in Example 1 .

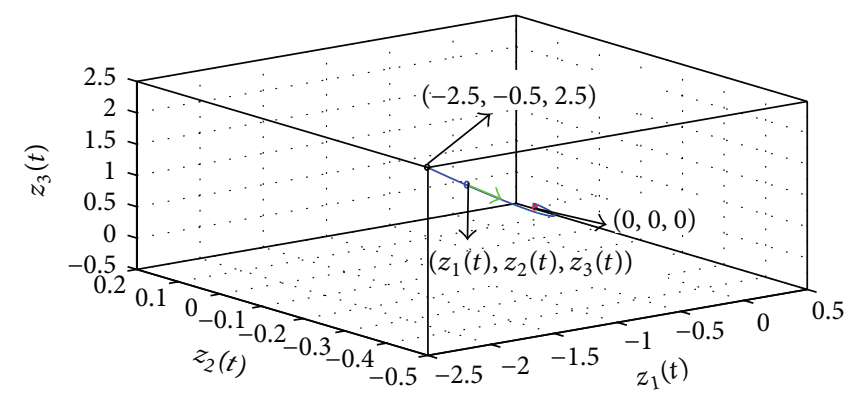

FIGURE 3: Phase trajectory of model of $z(t)$ in the space for $h=9.8356$ in Example 1 .

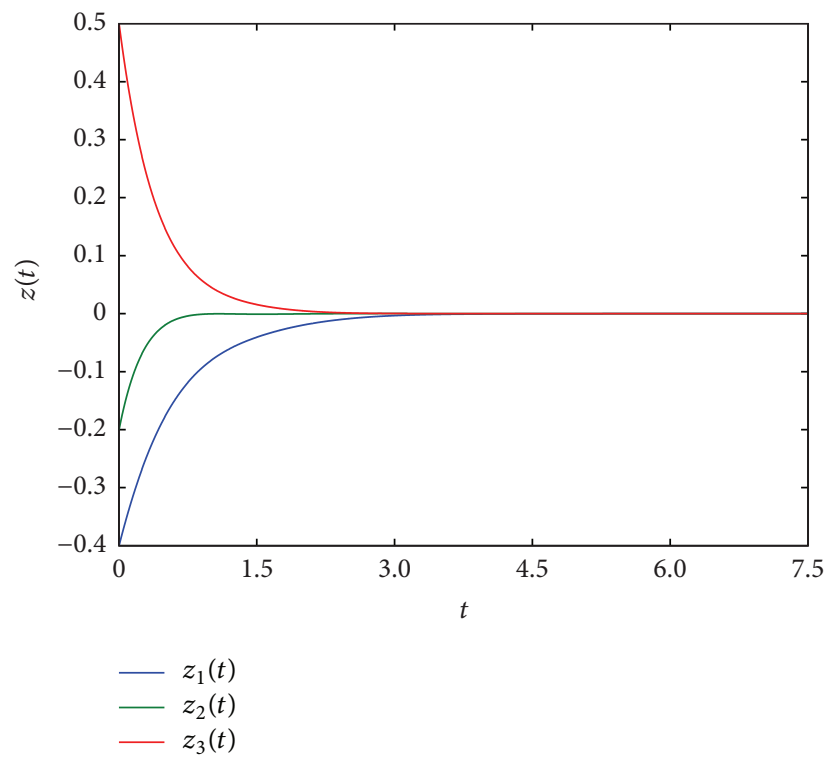

FIGURE 4: State trajectories of $z(t)$ in the plane for $h=5.220$ in Example 2. 


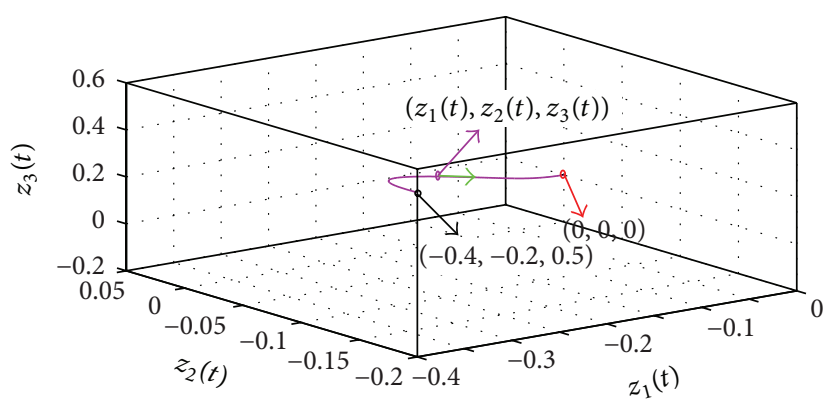

Figure 5: Phase trajectory of model of $z(t)$ in the plane for $h=5.220$ in Example 2.

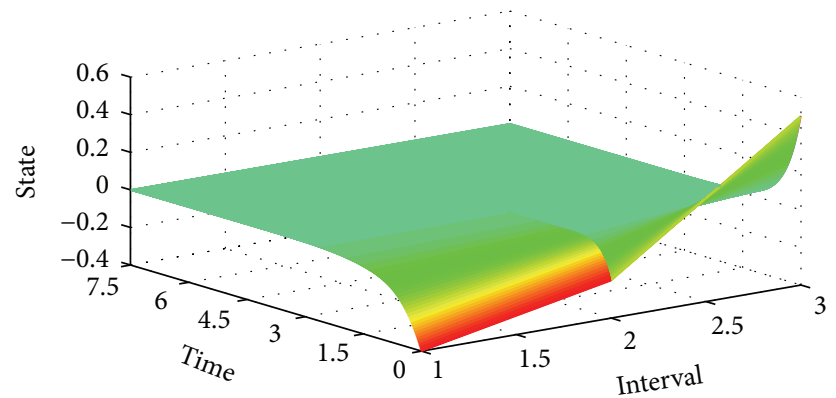

Figure 6: State trajectories of $z(t)$ on the space for $h=5.220$ in Example 2.

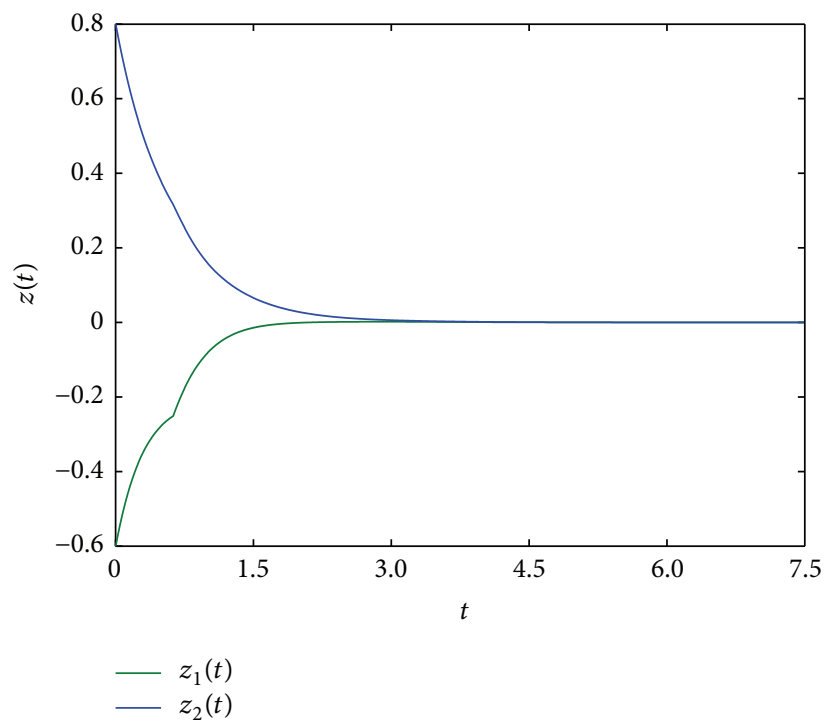

FIGURE 7: State trajectories of $z(t)$ in the plane for $h=3.518$ in Example 3.

\section{Conclusions}

In this paper, the delay-dependent exponential stability problem for NNs with mixed time-varying delays has been investigated. By using a new integral inequality approach to express the relationship between the terms in the LeibnizNewton formula within the framework of LMIs for the first time, several less conservative delay-dependent exponential stability criteria are obtained. Moreover, combining effective mathematical techniques and a convex optimization approach, new delay-dependent exponential stability conditions are derived by constructing a proper LKF. Finally, three numerical examples are given to illustrate the feasibility and effectiveness of the proposed methods. The foregoing 

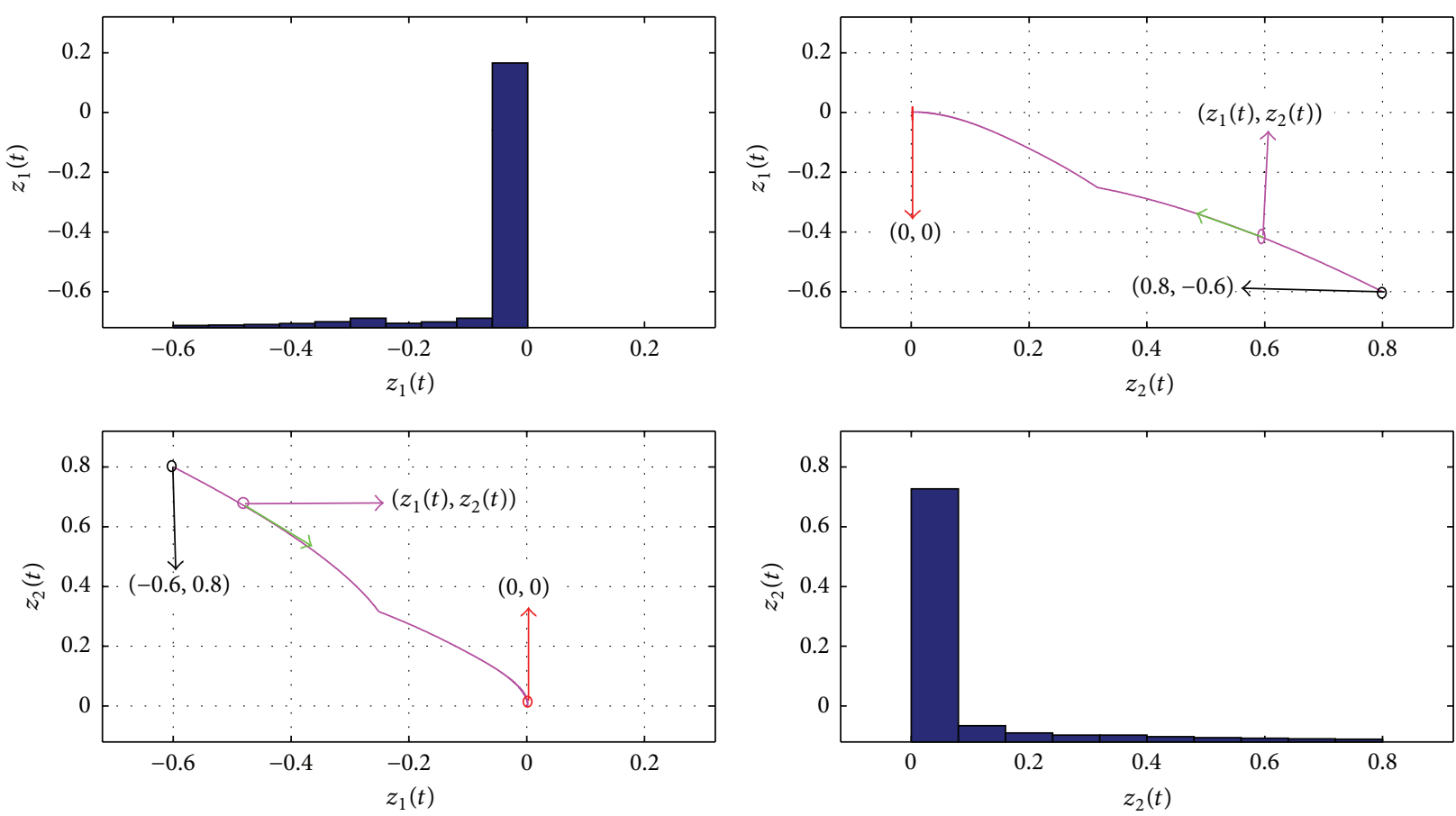

FIGURE 8: Phase trajectory of model of $z(t)$ in the plane for $h=3.518$ in Example 3.

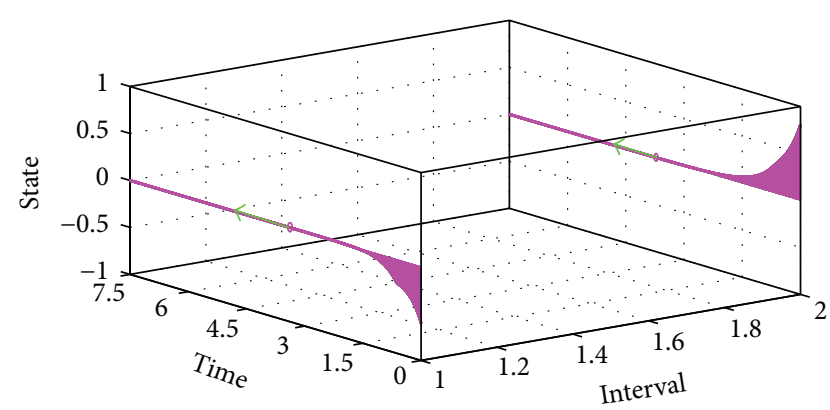

FIGURE 9: State trajectories of $z(t)$ on the space for $h=3.518$ in Example 3.

methods have the potential to be useful for the further study of neural network. Meanwhile, it is expected that these approaches can be further applied to other delayed systems.

\section{Disclaimer}

The authors promise that this paper has not been published and will not be simultaneously submitted or published elsewhere.

\section{Conflict of Interests}

The authors declare that there is no conflict of interests regarding the publication of this paper.

\section{Acknowledgments}

This work was supported by National Basic Research Program of China (2010CB732501), National Natural Science Foundation of China (61273015), the National Defense Pre-Research Foundation of China (Grant no. 9140A27040213DZ02001), the Fundamental Research Funds for the Central Universities (ZYGX2014J070), and the Program for New Century Excellent Talents in University (NCET-10-0097).

\section{References}

[1] F. Beaufays, Y. Abdel-Magid, and B. Widrow, "Application of neural networks to load-frequency control in power systems," Neural Networks, vol. 7, no. 1, pp. 183-194, 1994.

[2] M. Galicki, H. Witte, J. Dörschel, M. Eiselt, and G. Griessbach, "Common optimization of adaptive preprocessing units and a neural network during the learning period. Application in EEG pattern recognition," Neural Networks, vol. 10, no. 6, pp. 1153$1163,1997$.

[3] J. W. Cao and Z. P. Lin, "Bayesian signal detection with compressed measurements," Information Sciences, vol. 289, pp. 241-253, 2014.

[4] J. Cao, T. Chen, and J. Fan, "Landmark recognition with compact BoW histogram and ensemble ELM," Multimedia Tools and Applications, 2015.

[5] J. Cao and L. Xiong, "Protein sequence classification with improved extreme learning machine algorithms," BioMed Research International, vol. 2014, Article ID 103054, 12 pages, 2014. 
[6] J. W. Cao, "Improved delay-dependent stability conditions for MIMO networked control systems with nonlinear perturbations," The Scientific World Journal, vol. 2014, Article ID 196927, 4 pages, 2014.

[7] J. Cao, T. Chen, and J. Fan, "Fast online learning algorithm for landmark recognition based on BoW framework," in Proceedings of the 9th IEEE Conference on Industrial Electronics and Applications (ICIEA '14), pp. 1163-1168, Hangzhou, China, June 2014.

[8] J. K. Tian, W. J. Xiong, and F. Xu, "Improved delay-partitioning method to stability analysis for neural networks with discrete and distributed time-varying delays," Applied Mathematics and Computation, vol. 233, pp. 152-164, 2014.

[9] J. Cheng, S. M. Zhong, Q. S. Zhong, H. Zhu, and Y. H. $\mathrm{Du}$, "Finite-time boundedness of state estimation for neural networks with time-varying delays," Neurocomputing, vol. 129, pp. 257-264, 2014.

[10] D. Zhang and L. Yu, "Exponential state estimation for Markovian jumping neural networks with time-varying discrete and distributed delays," Neural Networks, vol. 35, pp. 103-111, 2012.

[11] J. H. Park and O. M. Kwon, "Synchronization of neural networks of neutral type with stochastic perturbation," Modern Physics Letters B, vol. 23, no. 14, pp. 1743-1751, 2009.

[12] X. Li and J. Cao, "Delay-dependent stability of neural networks of neutral type with time delay in the leakage term," Nonlinearity, vol. 23, no. 7, pp. 1709-1726, 2010.

[13] M. Luo, S. Zhong, R. Wang, and W. Kang, "Robust stability analysis for discrete-time stochastic neural networks systems with time-varying delays," Applied Mathematics and Computation, vol. 209, no. 2, pp. 305-313, 2009.

[14] J. W. Xia, J. Y. Yu, Y. M. Li, and H. Zheng, "New delay-intervaldependent exponential stability for stochastic neural networks with interval time-varying delay and distributed delay," Circuits, Systems, and Signal Processing, vol. 31, no. 4, pp. 1535-1557, 2012.

[15] S. Lakshmanan and P. Balasubramaniam, "New results of robust stability analysis for neutral-type neural networks with timevarying delays and Markovian jumping parameters," Canadian Journal of Physics, vol. 89, no. 8, pp. 827-840, 2011.

[16] O. M. Kwon, J. H. Park, S. M. Lee, and E. J. Cha, "A new augmented Lyapunov-Krasovskii functional approach to exponential passivity for neural networks with time-varying delays," Applied Mathematics and Computation, vol. 217, no. 24, pp. 10231-10238, 2011.

[17] P.-L. Liu, "Improved delay-dependent robust stability criteria for recurrent neural networks with time-varying delays," ISA Transactions, vol. 52, no. 1, pp. 30-35, 2013.

[18] C.-H. Lien and L.-Y. Chung, "Global asymptotic stability for cellular neural networks with discrete and distributed timevarying delays," Chaos, Solitons \& Fractals, vol. 34, no. 4, pp. 1213-1219, 2007.

[19] Q. K. Song and Z. D. Wang, "Neural networks with discrete and distributed time-varying delays: a general stability analysis," Chaos, Solitons \& Fractals, vol. 37, no. 5, pp. 1538-1547, 2008.

[20] T. Li, Q. Luo, C. Sun, and B. Zhang, "Exponential stability of recurrent neural networks with time-varying discrete and distributed delays," Nonlinear Analysis. Real World Applications, vol. 10, no. 4, pp. 2581-2589, 2009.

[21] X. Zhu and Y. Wang, "Delay-dependent exponential stability for neural networks with discrete and distributed time-varying delays," Physics Letters A, vol. 373, no. 44, pp. 4066-4072, 2009.
[22] J. K. Tian and S. Zhong, "New delay-dependent exponential stability criteria for neural networks with discrete and distributed time-varying delays," Neurocomputing, vol. 74, no. 17, pp. 33653375, 2011.

[23] J. Tian, S. Zhong, and Y. Wang, "Improved exponential stability criteria for neural networks with time-varying delays," Neurocomputing, vol. 97, pp. 164-173, 2012.

[24] L. Shi, H. Zhu, S. Zhong, and L. Y. Hou, "Globally exponential stability for neural networks with time-varying delays," Applied Mathematics and Computation, vol. 219, no. 21, pp. 10487-10498, 2013.

[25] J.-D. Cao, "Global asymptotic stability of neural networks with transmission delays," International Journal of Systems Science, vol. 31, no. 10, pp. 1313-1316, 2010.

[26] J. Cao and J. Wang, "Global asymptotic stability of a general class of recurrent neural networks with time-varying delays," IEEE Transactions on Circuits and Systems I, vol. 50, no. 1, pp. 34-44, 2003.

[27] J. Cao and T. Chen, "Globally exponentially robust stability and periodicity of delayed neural networks," Chaos, Solitons and Fractals, vol. 22, no. 4, pp. 957-963, 2004.

[28] J. Cao and J. Wang, "Global asymptotic and robust stability of recurrent neural networks with time delays," IEEE Transactions on Circuits and Systems. I. Regular Papers, vol. 52, no. 2, pp. 417426, 2005.

[29] Y. L. Wang and J. D. Cao, "Exponential stability of stochastic higher-order BAM neural networks with reaction-diffusion terms and mixed time-varying delays," Neurocomputing, vol. 119, pp. 192-200, 2013.

[30] J. D. Cao and Y. Wan, "Matrix measure strategies for stability and synchronization of inertial BAM neural network with time delays," Neural Networks, vol. 53, pp. 165-172, 2014.

[31] Z. Zhang, J. Cao, and D. Zhou, "Novel LMI-based condition on global asymptotic stability for a class of cohen-grossberg BAM networks with extended activation functions," IEEE Transactions on Neural Networks and Learning Systems, vol. 25, no. 6, pp. 1161-1172, 2014.

[32] K. B. Shi, H. Zhu, S. M. Zhong, Y. Zeng, and Y. P. Zhang, "Improved delay-dependent stability criteria for neural networks with discrete and distributed time-varying delays using a delay-partitioning approach," Nonlinear Dynamics, vol. 79, no. 1, pp. 575-592, 2015.

[33] K. B. Shi, H. Zhu, S. M. Zhong, Y. Zeng, and Y. Zhang, "New stability analysis for neutral type neural networks with discrete and distributed delays using a multiple integral approach," Journal of the Franklin Institute, vol. 352, no. 1, pp. 155-176, 2015.

[34] S. Fang, M. Jiang, and X. Wang, "Exponential convergence estimates for neural networks with discrete and distributed delays," Nonlinear Analysis: Real World Applications, vol. 10, no. 2, pp. 702-714, 2009.

[35] H. Li, B. Chen, Q. Zhou, and S. Fang, "Robust exponential stability for uncertain stochastic neural networks with discrete and distributed time-varying delays," Physics Letters A, vol. 372, no. 19, pp. 3385-3394, 2008.

[36] L. Ma and F. Da, "Mean-square exponential stability of stochastic Hopfield neural networks with time-varying discrete and distributed delays," Physics Letters A, vol. 373, no. 25, pp. 21542161, 2009.

[37] K. B. Shi, H. Zhu, S. M. Zhong, Y. Zeng, and Y. P. Zhang, "Less conservative stability criteria for neural networks with discrete and distributed delays using a delay-partitioning approach," Neurocomputing, vol. 140, pp. 273-282, 2014. 
[38] Z.-G. Wu, P. Shi, H. Su, and J. Chu, "Passivity analysis for discrete-time stochastic markovian jump neural networks with mixed time delays," IEEE Transactions on Neural Networks, vol. 22, no. 10, pp. 1566-1575, 2011.

[39] D. Zhang, L. Yu, Q.-G. Wang, and C.-J. Ong, "Estimator design for discrete-time switched neural networks with asynchronous switching and time-varying delay," IEEE Transactions on Neural Networks and Learning Systems, vol. 23, no. 5, pp. 827-834, 2012.

[40] Z.-G. Wu, J. H. Park, H. Su, and J. Chu, "New results on exponential passivity of neural networks with time-varying delays," Nonlinear Analysis: Real World Applications, vol. 13, no. 4, pp. 1593-1599, 2012.

[41] X. B. Zhou, J. K. Tian, H. J. Ma, and S. M. Zhong, "Improved delay-dependent stability criteria for recurrent neural networks with time-varying delays," Neurocomputing, vol. 129, pp. 401408, 2014

[42] Z.-G. Wu, P. Shi, H. Y. Su, and J. Chu, "Exponential synchronization of neural networks with discrete and distributed delays under time-varying sampling," IEEE Transactions on Neural Networks and Learning Systems, vol. 23, no. 9, pp. 1368-1376, 2012.

[43] C. Yin, S.-M. Zhong, and W.-F. Chen, "Robust $H_{\infty}$ control for uncertain Lure systems with sector and slope restricted nonlinearities by PD state feedback," Nonlinear Analysis: Real World Applications, vol. 12, no. 1, pp. 501-512, 2011.

[44] C. Yin, Y. Q. Chen, and S. M. Zhong, "Fractional-order sliding mode based extremum seeking control of a class of nonlinear systems," Automatica, vol. 50, no. 12, pp. 3173-3181, 2014.

[45] J. Cheng and L. L. Xiong, "Improved integral inequality approach on stabilization for continuous-time systems with time-varying input delay," Neurocomputing, vol. 160, pp. 274280, 2015.

[46] J. Cheng, L. Xiong, B. Wang, and J. Yang, "Robust finite-time boundedness of $H_{\infty}$ filtering for switched systems with timevarying delay," Optimal Control Applications and Methods, 2015.

[47] J. Cheng, H. Zhu, S. Zhong, Q. Zhong, and Y. Zeng, "Finitetime $H_{\infty}$ estimation for discrete-time Markov jump systems with time-varying transition probabilities subject to average dwell time switching," Communications in Nonlinear Science and Numerical Simulation, vol. 20, no. 2, pp. 571-582, 2015.

[48] X. D. Li and X. L. Fu, "Effect of leakage time-varying delay on stability of nonlinear differential systems," Journal of the Franklin Institute, vol. 350, no. 6, pp. 1335-1344, 2013.

[49] X. Li and M. Bohner, "An impulsive delay differential inequality and applications," Computers \& Mathematics with Applications, vol. 64, no. 6, pp. 1875-1881, 2012.

[50] X. D. Li and R. Rakkiyappan, "Impulsive controller design for exponential synchronization of chaotic neural networks with mixed delays," Communications in Nonlinear Science and Numerical Simulation, vol. 18, no. 6, pp. 1515-1523, 2013.

[51] X. D. Li and S. J. Song, "Impulsive control for existence, uniqueness, and global stability of periodic solutions of recurrent neural networks with discrete and continuously distributed delays," IEEE Transactions on Neural Networks and Learning Systems, vol. 24, no. 6, pp. 868-877, 2013.

[52] X. D. Li and S. J. Song, "Research on synchronization of chaotic delayed neural networks with stochastic perturbation using impulsive control method," Communications in Nonlinear Science and Numerical Simulation, vol. 19, no. 10, pp. 3892-3900, 2014.

[53] K. Shi, H. Zhu, S. Zhong, Y. Zeng, Y. Zhang, and L. Liang, "Less conservative stability criteria for neutral type neural networks with mixed time-varying delays," Journal of Applied Mathematics, vol. 2013, Article ID 450175, 18 pages, 2013.

[54] P. Balasubramaniam, R. Krishnasamy, and R. Rakkiyappan, "Delay-dependent stability of neutral systems with timevarying delays using delay-decomposition approach," Applied Mathematical Modelling. Simulation and Computation for Engineering and Environmental Systems, vol. 36, no. 5, pp. 2253-2261, 2012. 


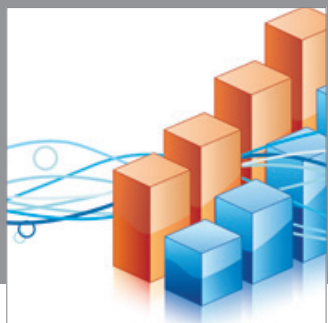

Advances in

Operations Research

mansans

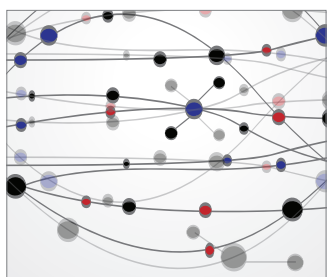

The Scientific World Journal
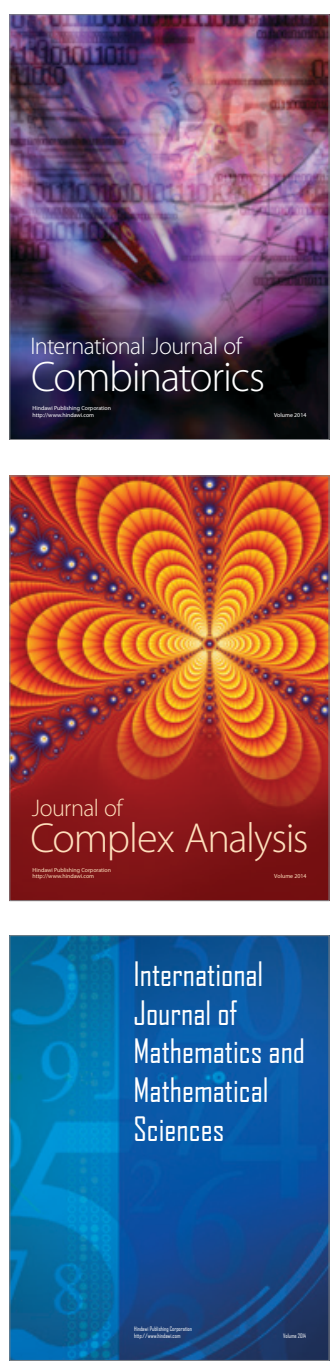
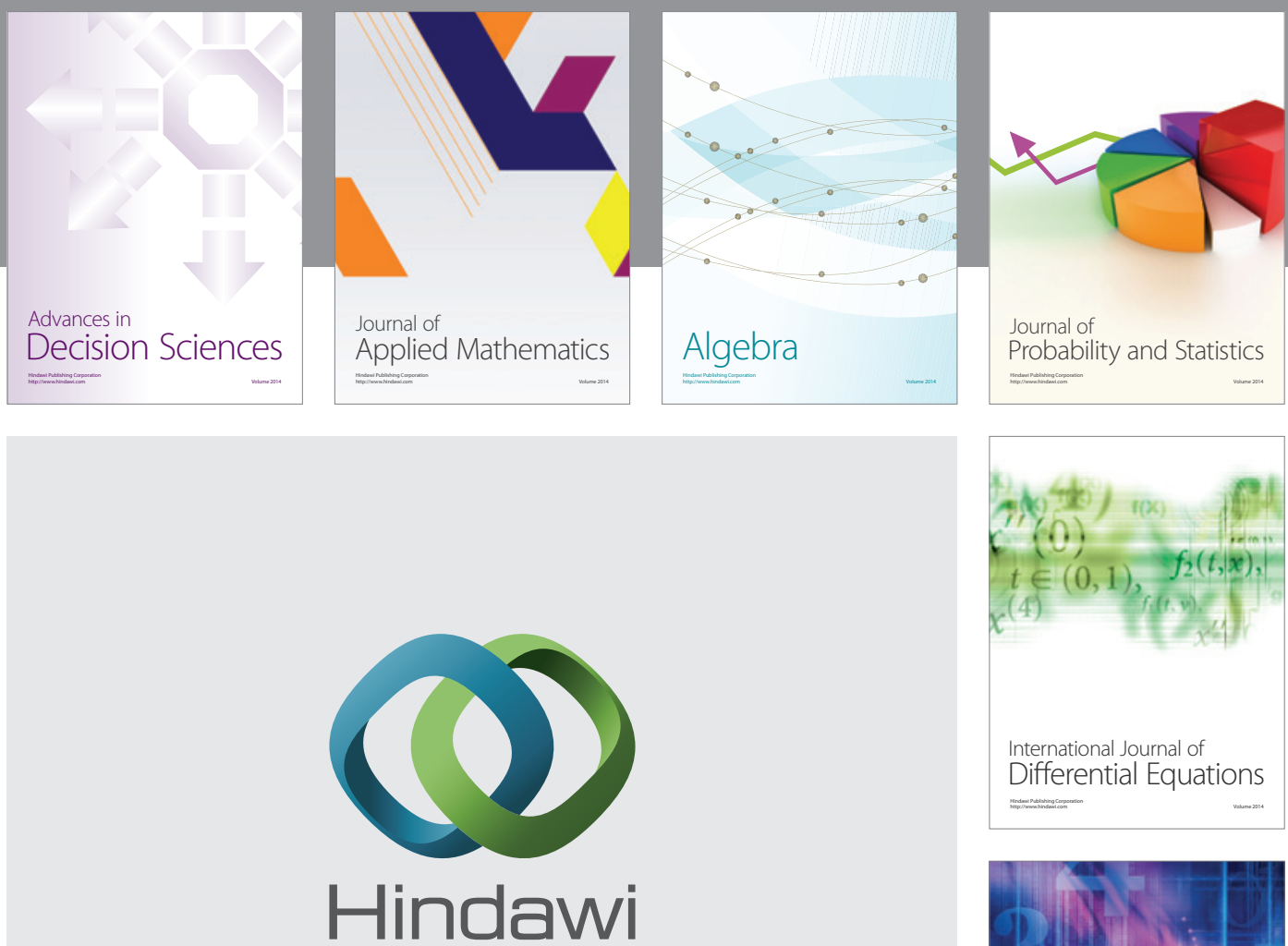

Submit your manuscripts at http://www.hindawi.com
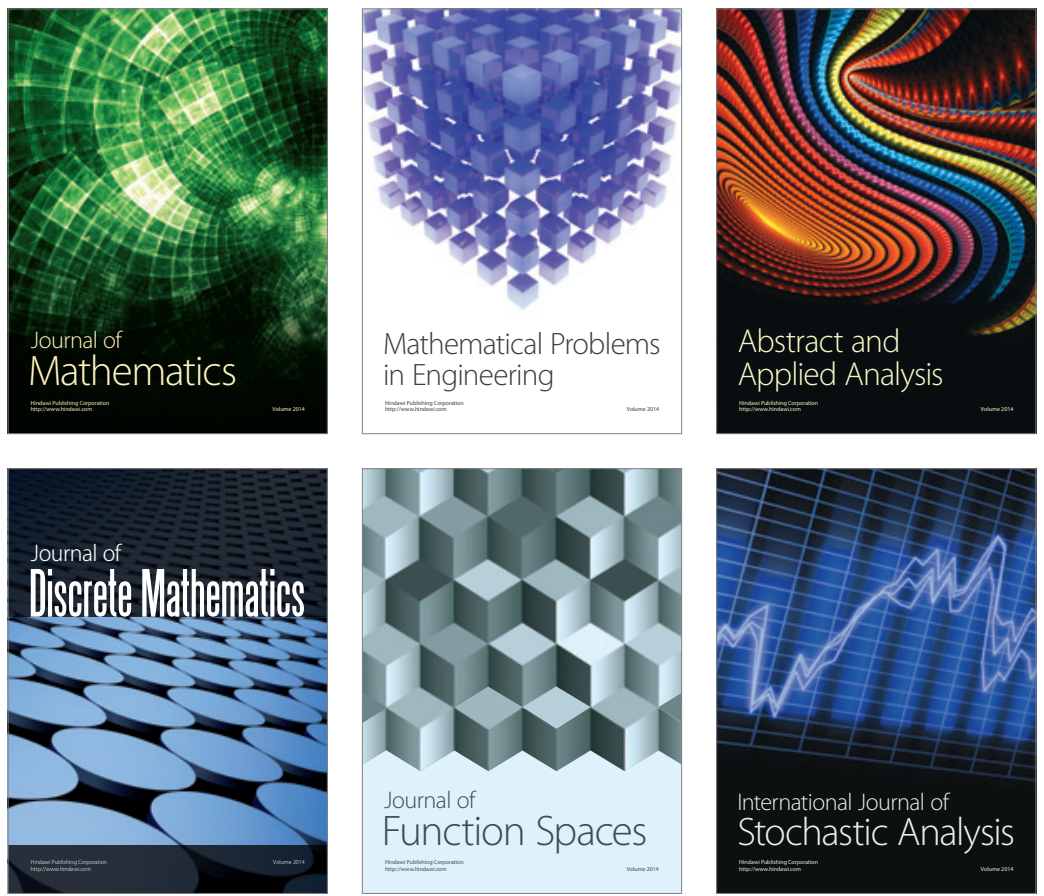

Journal of

Function Spaces

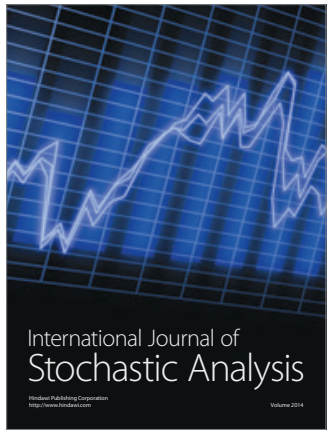

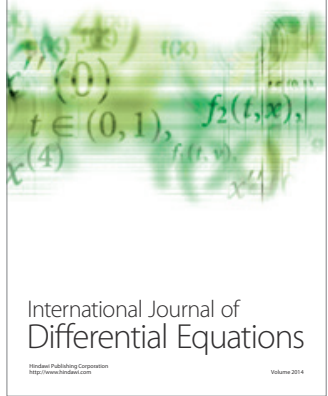
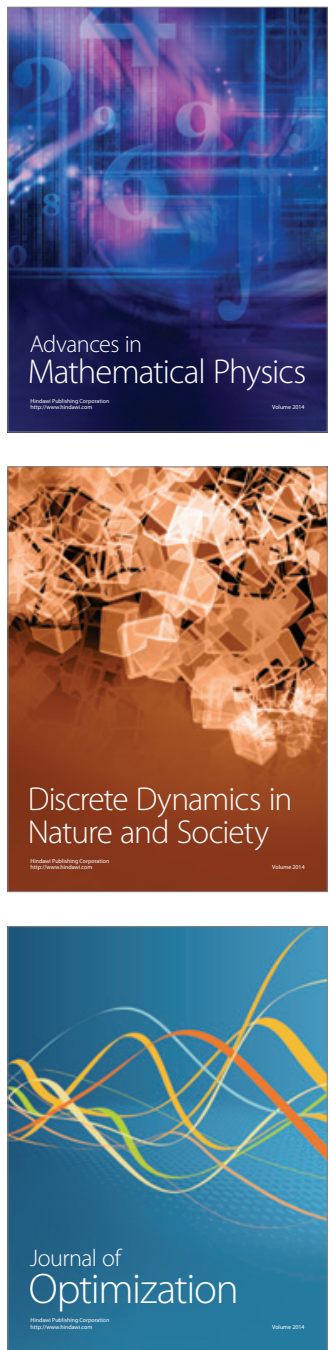